Mon. Not. R. Astron. Soc. 000, 1-?? (2003) Printed 23 June $2018 \quad$ (MN LATEX style file v2.2)

\title{
Resolving the Kinematic Distance Ambiguity of Southern Massive Young Stellar Object Candidates
}

\author{
A. L. Busfield,${ }^{1 \star}$ C. R. Purcell,${ }^{2} \dagger$ M. G. Hoare,${ }^{1 \star}$ S. L. Lumsden, ${ }^{1 \star}$ \\ T. J. T. Moore ${ }^{3} \ddagger$ and R. D. Oudmaijer ${ }^{1 \star}$ \\ ${ }^{1}$ School of Physics and Astronomy, University of Leeds, Leeds, LS2 9JT, UK \\ ${ }^{2}$ School of Physics, University of New South Wales, Sydney, Australia 2052 \\ ${ }^{3}$ Astrophysics Research Institute, Liverpool John Moores University, Twelve Quays House, Egerton Wharf, Birkenhead CH41 1LD, UK
}

Accepted Received

\begin{abstract}
We investigate the use of $\mathrm{H}$ I data to resolve the near/far ambiguity in kinematic distances of massive young stellar object (MYSO) candidates. Kinematic distances were obtained from ${ }^{13} \mathrm{CO} 1-0$ (and $\mathrm{N}_{2} \mathrm{H}^{+}$) spectral line observations with the Mopra Telescope towards 94 candidates selected from the Red MSX Source (RMS) survey in the fourth Galactic quadrant $\left(282^{\circ}<l<350^{\circ}\right)$. H I data from the Southern Galactic Plane Survey (SPGS) was used in conjunction with the H I self-absorption technique to determine the near or far distance. We resolved the kinematic distance ambiguity to $70 \%$ of the sources. We can also simultaneously solve for any multiple line-of-sight component sources. We discuss the advantages and disadvantages of this technique in comparison with other methods, and also perform confidence checks on the reliability of using the $\mathrm{H}$ I self-absorption technique.

We examined the projected location of these sources in both the Galactic plane and longitude-velocity diagrams to ascertain any recognisable spiral arm pattern. Although no obvious spiral pattern was found when compared to that proposed by Cordes \& Lazio, far distance sources tended to lie on or near spiral arm loci. Near distance sources, however, had peculiar velocity uncertainties consistent with the separation between the spiral arms themselves. The longitude-velocity plot shows a more consistent picture, with tangent points of the spiral arm loci easily seen.

We conclude that using the $\mathrm{H}$ I self-absorption technique to determine kinematic distance ambiguities is a quick and reliable method in most cases, with an $80 \%$ success rate in determining the correct designation of whether an object is at the near or far distance.
\end{abstract}

Key words: ISM: Galaxy : structure, kinematics and dynamics.

\section{INTRODUCTION}

Stars with mass $>8 \mathrm{M}_{\odot}\left(\mathrm{L}>10^{4} \mathrm{~L}_{\odot}\right)$, play important roles in many areas of astrophysics. They are fundamental to the evolution of galaxies, because they produce the heavy elements, inject large amounts of UV radiation, wind energy and strong shocks into the interstellar medium throughout their short lifetimes, and possibly regulate the star formation rate. Even though much has been learnt over the past two decades about the evolution of massive stars, their birth still remains mysterious.

\footnotetext{
* E-mail: alb,mgh,sll,roud@ast.leeds.ac.uk

$\dagger$ E-mail: crp@unsw.edu.au

$\ddagger$ E-mail: tjtm@astro.livjm.ac.uk
}

Many aspects of the study of massive star formation suffer from the lack of a large unbiased sample. In particular the observationally known sample of massive young stellar objects (MYSOs) only consists of thirty or so well catalogued objects (Henning et al. 1984). Formed just after the hot core phase, MYSOs are mid-IR bright objects where fusion has probably begun within the core, however, it has not yet started to ionise the surroundings to form an ultracompact H II region. Many of the known MYSOs are nearby and may not represent this class of objects as a whole. Hence the need for finding a larger sample of MYSOs within our Galaxy, using an unbiased selection technique.

We have developed colour-selection criteria that deliver about 2000 candidate MYSOs from the $M S X$ point source catalogue with the addition of 2MASS data Lumsden et al. 
2002). There are many other types of source which potentially have the same very red colours as MYSOs, especially compact H II regions, but also some planetary nebulae, postAGB stars, dusty red supergiants and low mass YSOs. A large follow-up programme, the Red MSX Source or RMS Survey (Hoare et al. 2004) is underway to identify and eliminate these other sources from the sample. A key part of the follow-up is the determination of distances to these sources in order to obtain bolometric luminosities. In fact, some of them might be low luminosity YSOs and therefore should be removed from the sample.

The determination of kinematic distances only requires the measurement of the radial velocity of the source together with the application of a specific Galactic rotation curve (e.g. Brand \& Blitz 1993). Although the determination of kinematic distances in the outer Galaxy is relatively trivial, it becomes much more difficult towards the inner Galaxy due to the distance ambiguity problem. In the inner Galaxy a single radial velocity value relates to two distances (a near and far distance) either side of the tangent point, along any one line of sight within the solar circle. Only sources that lie at the tangent point have unambiguous distances.

Many molecular line studies have been used to search for massive young stars, dense molecular cores or H II regions, and determine their distances (Downes et al. 1980; Wink. Altenhoff \& Mezgen 1982; Wu. Wu \& Wang 2001). However, the majority of these studies have side-stepped the distance ambiguity problem altogether by observing sources outside the solar circle (e.g. Wouterloot \& Brand 1989) hence ignoring the inner Galaxy where the bulk of massive star formation occurs. A number of studies where inner Galaxy objects have been observed have either been left with the unsolved ambiguity problem and no kinematic distances calculated (e.g. Sridharan et al. 2002), or have assumed all objects with an ambiguity to be at a near or a far distance, resulting in bolometric luminosities which can be orders of magnitude different (e.g. Wu, Wu \& Wang 2001).

Several studies have tried to solve the kinematic distance ambiguity to a small number of sources using various techniques. Downes et al. (1980); Arava et al. (2001, 2002) have combined measurements of the $\mathrm{H} 110 \alpha$ radio recombination line (RRL) and the $\mathrm{H}_{2} \mathrm{CO}$ (formaldehyde) absorption line to distinguish between near and far distances. To determine kinematic distances using this method, it is assumed that all $\mathrm{H}_{2} \mathrm{CO}$ absorption lines are produced by absorption of radio continuum from a single background $\mathrm{H}$ II region. Based on these assumptions, if the $\mathrm{H}_{2} \mathrm{CO}$ absorption occurs at higher velocities than the RRL, then the $\mathrm{H}$ II region has to be located at the far distance. If no molecular gas is detected at velocities higher than the RRL, then the near kinematic distance is implied for the H II region. Although this method is useful, it can only be used for radio bright sources, whereas the MYSOs are known to be radio quiet.

A more statistical approach was made by Paladini. Davies \& DeZotti (2004) using the luminosityphysical diameter correlation of $\mathrm{H}$ II regions. The relationship is based on the argument that the luminosity is proportional to the product of the emission measure (which is proportional to the linear diameter) with the square of the angular size. A highly significant relationship was found, and resolved the distance ambiguity to 256 sources. However, this method is again only able to be used for well

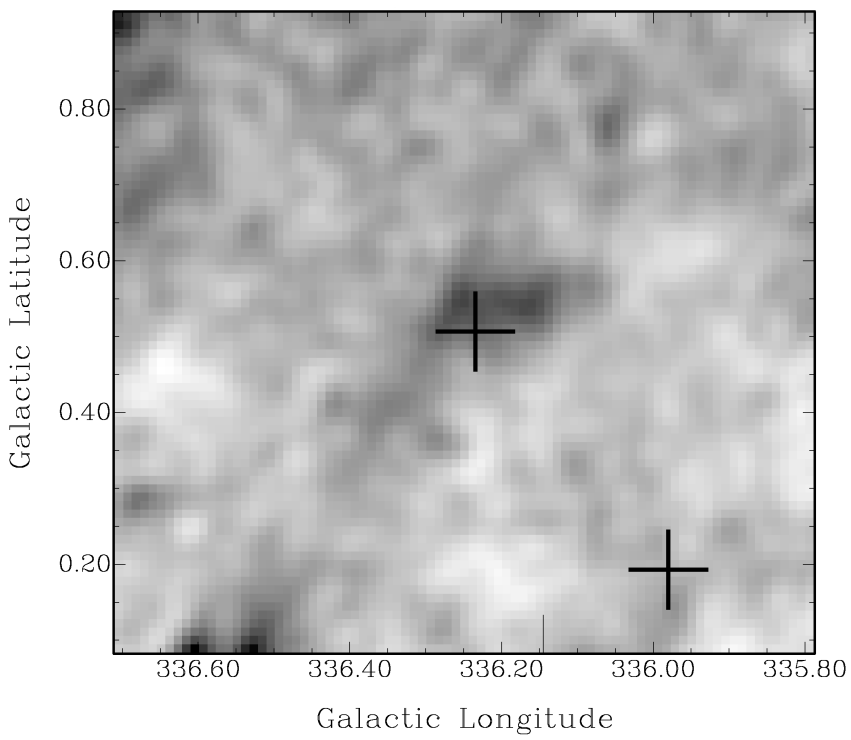

Figure 1. An example H i cube slice (greyscale) centered at the $v=-29.7 \mathrm{~km} \mathrm{~s}^{-1}$ velocity channel overlaid with the central position of one of our MSX targets G336.2344+00.5067, showing a slightly extended darker (absorption) region around the object. The other black cross indicates another RMS potential MYSO candidate within the field at a different velocity.

behaved $\mathrm{H}$ II regions, and is only successful in solving $60 \%$ of re-tested cases where the distance ambiguity had already been resolved.

Burton, Liszt \& Baker 1978) and Liszt, Burton \& Bania (1981) (hereafter LBB) identified several H I self-absorption (SA) features in $21 \mathrm{~cm} \mathrm{H} \mathrm{I}$ maps made of the Galactic plane and noted that many of these also correlate with $\mathrm{CO}$ emission features. LBB hypothesised that H I SA may provide a robust method for determining the distance to molecular clouds. If the cold $\mathrm{HI}$ in a molecular cloud absorbs warmer $\mathrm{HI}$ background line emission, then clouds at the near distance should show H I SA, whereas clouds at the far distance will not since there there is no background to absorb. Jackson et al. (2002) solved the distance ambiguity to the molecular cloud GRSMC 45.6+0.3 using H I self-absorption (SA). This approach requires all molecular clouds have to contain sufficient cold atomic hydrogen in order for $\mathrm{H}$ I SA to occur. Although this still needs to be confirmed observationally, Flynn et al. (2004) have shown that all their model molecular clouds contain enough opacity in cold $\mathrm{H}$ I to exhibit self-absorption against strong $21 \mathrm{~cm}$ backgrounds.

Up to now, H I data with spatial resolution comparable to CO surveys and covering the whole Galaxy were not available, with only small samples of the Galactic plane surveyed such as the Boston University-Arecibo Observatory (BUAO) Galactic H I Survey (Kuchar \& Bania 1993). These data have since been successfully used with the $\mathrm{HI}$ SA method on a small selection of objects by Kolpak et al. (2003). However, with the near completion of the International Galactic Plane Survey (IGPS), it is now possible to use this method to study the entire Galaxy. Further details about this survey will be given in the next section.

Many of our candidate MYSOs have now been observed 
at ${ }^{13} \mathrm{CO} 1-0$ to obtain kinematic distances and hence bolometric luminosities. Unlike ${ }^{12} \mathrm{CO} 1-0,{ }^{13} \mathrm{CO} 1-0$ has narrower line widths allowing much better separation of the velocity components. Furthermore it also avoids some of the difficulties of self-absorbed line profiles often found with ${ }^{12} \mathrm{CO}$ data especially towards the inner Galaxy reducing the assignment of an incorrect spectral line velocity.

The source list was compiled primarily from the second release of the $M S X$ point source catalogue, using colourselection criteria for MYSOs from Lumsden et al. (2002) combined with visual inspection of all the $M S X$ images for likely targets, delivering 2000 objects. This paper will concentrate on a sample of these objects with a distance ambiguity in the 4th Galactic quadrant and it will discuss whether the Hi SA technique is a valid method for determining their kinematic distance.

\section{OBSERVATIONS AND DATA REDUCTION}

The ${ }^{13}$ CO 1-0 observations were carried out in the periods of August 2002 and July 2003, using the Mopra 22-m millimetre-wave telescope of the ATNF. The assumed rest frequency of the ${ }^{13} \mathrm{CO} 1-0$ transition was $110.201353 \mathrm{GHz}$. The half-power beamwidth of the Mopra antenna at ${ }^{13} \mathrm{CO}$ $1-0$ is $\sim 35^{\prime \prime}$. The back-end was a $64 \mathrm{MHz}$ wide 1024-channel autocorrelator, which yields a channel resolution at $110 \mathrm{GHz}$ of $0.17 \mathrm{~km} \mathrm{~s}^{-1}$.

The observations were performed in a positionswitching mode with reference positions offset 1 degree away from the object, in a direction away from the plane of the Galaxy. Total on-source time was usually 10 minutes, using $10 \times 1$ minute integrations; less if the lines were strong, giving a rms noise of $\sim 0.1 \mathrm{~K}$. The reference position was chosen to try and minimise any off-beam emission from appearing at the on-source velocity within the crowded Galactic plane. The antenna pointing was checked and corrected every 2-3 hours by making observations of strong $86 \mathrm{GHz} \mathrm{SiO}$ masers; the pointing accuracy when this procedure is followed is $\sim 10$ arcsec.

A cryogenically cooled low-noise SIS mixer was used in the receiver. The system temperature varied between 240 and $400 \mathrm{~K}$, depending on weather conditions and the elevation of the telescope. Although we were only primarily interested in obtaining a kinematic velocity, and not accurate fluxes, an ambient temperature load (assumed to have a temperature of $290 \mathrm{~K}$ ) was regularly placed in front of the receiver to enable calibration of the observed flux density for the effects of atmospheric attenuation. The estimated uncertainty of the absolute flux density scale is 20 per cent.

The ${ }^{13} \mathrm{CO} 1-0$ spectral line data were reduced in the standard way with DFM (Data From Mopra) ${ }^{1}$. The separate integrations were averaged, and baseline subtracted. To improve the signal-to-noise ratio of weak sources, the spectra were then Hanning smoothed. Gaussians were fitted to all line components, and any significant off-beam sources that do not affect the line profile zeroed. The uncertainty in fitting the velocity profiles was typically $\pm 0.1 \mathrm{~km}^{-1}$

1 A tcl/tk graphical interface written for SPC (Spectral Line Reduction Package), by C. Purcell.
Where there are multiple ${ }^{13} \mathrm{CO}$ emission components along a single line of sight, we have identified the likely MYSO component to be the strongest and/or widest component within the spectra. However, this may not always be correct. If there is no broad component more than three times the strength of the others, the line with a width greater than $5 \mathrm{~km} \mathrm{~s}^{-1}$ was used. It should be noted that not all of these objects will be star-forming regions and the RMS survey is currently underway, identifying all these regions.

The IGPS is a collaboration of the Southern (SGPS) (McClure-Griffiths et al. 2001b), VLA (VGPS) (Tavlor et al. 2002), and Canadian (CGPS) (English et al. 1998) Galactic Plane Surveys. The H I data cubes were obtained from the Southern Galactic Plane Survey of McClure-Griffiths et al. (2001b). The SGPS consisted of 2212 mosaic pointings over a total area of 210 square degrees, with an angular resolution of $2^{\prime}$ and a rms line noise level of $\sim 2.3 \mathrm{~K}$. The continuum-subtracted cubes were viewed using the KARMA ${ }^{2}$ general-purpose image display software. The spectrum from the relevant object pixel position were extracted from the $\mathrm{H}$ I data cubes (Figure 1), with a pixel size of $\sim 0.02^{\circ}$. There were no significant spectral deviations from the central object pixel and its surroundings on any of the $\mathrm{H}$ i spectra.

For five of the RMS survey objects we use the $\mathrm{N}_{2} \mathrm{H}^{+}$ transition line instead of the ${ }^{13} \mathrm{CO} 1-0$ line to determine the kinematic distance, using data obtained in a similar way at Mopra by Purcell et al (in prep.). These RMS survey objects are sites of methanol maser emission, thought to be tracers of massive star formation. However, due to the spread in maser velocities (up to $\sim 10 \mathrm{~km} \mathrm{~s}^{-1}$ ) we are using the thermal emission line to obtain the object velocity. Due to hyperfine splitting of $\mathrm{N}_{2} \mathrm{H}^{+}$line, the position of the central peak was used to determine the velocity.

\section{RESULTS AND DISCUSSION}

Figure 2 shows detailed examples of the various types of ${ }^{13} \mathrm{CO}$ and overlaid $\mathrm{H}$ I spectral profiles that were obtained. Figure 2 (a) shows a clear near distance solution with the ${ }^{13} \mathrm{CO}$ emission line coinciding with a $\mathrm{H}$ I absorption. Figure 2 (b) represents a far distance object with no H I SA located at the ${ }^{13} \mathrm{CO}$ peak velocity, whereas Figure 2 (c) shows an example of an ambiguity, as overall the ${ }^{13} \mathrm{CO}$ peak lies in a $\mathrm{H}$ I trough, but within the trough is a spike, hence not providing any clear cut answer to the kinematic distance ambiguity.

The majority $(70 \%)$ of the distance ambiguity cases had spectral profiles indicating a clear near or far distance solution. Of the 94 sources, 33 of these are now determined to be at the near distance, and 33 objects at the far distance. A futher 24 objects had line features (described below) that were unable to provide a definite answer to the distance ambiguity, and so were designated a probable (best) solution. The distance ambiguities to 4 objects were unable to be solved by using this method.

The results of the Gaussian fits and resulting kinematic

2 KARMA is a toolkit for interprocess communications, authentication, encryption, graphics display, user interface and manipulating the KARMA network data structure, written by $\mathrm{R}$. Gooch. 


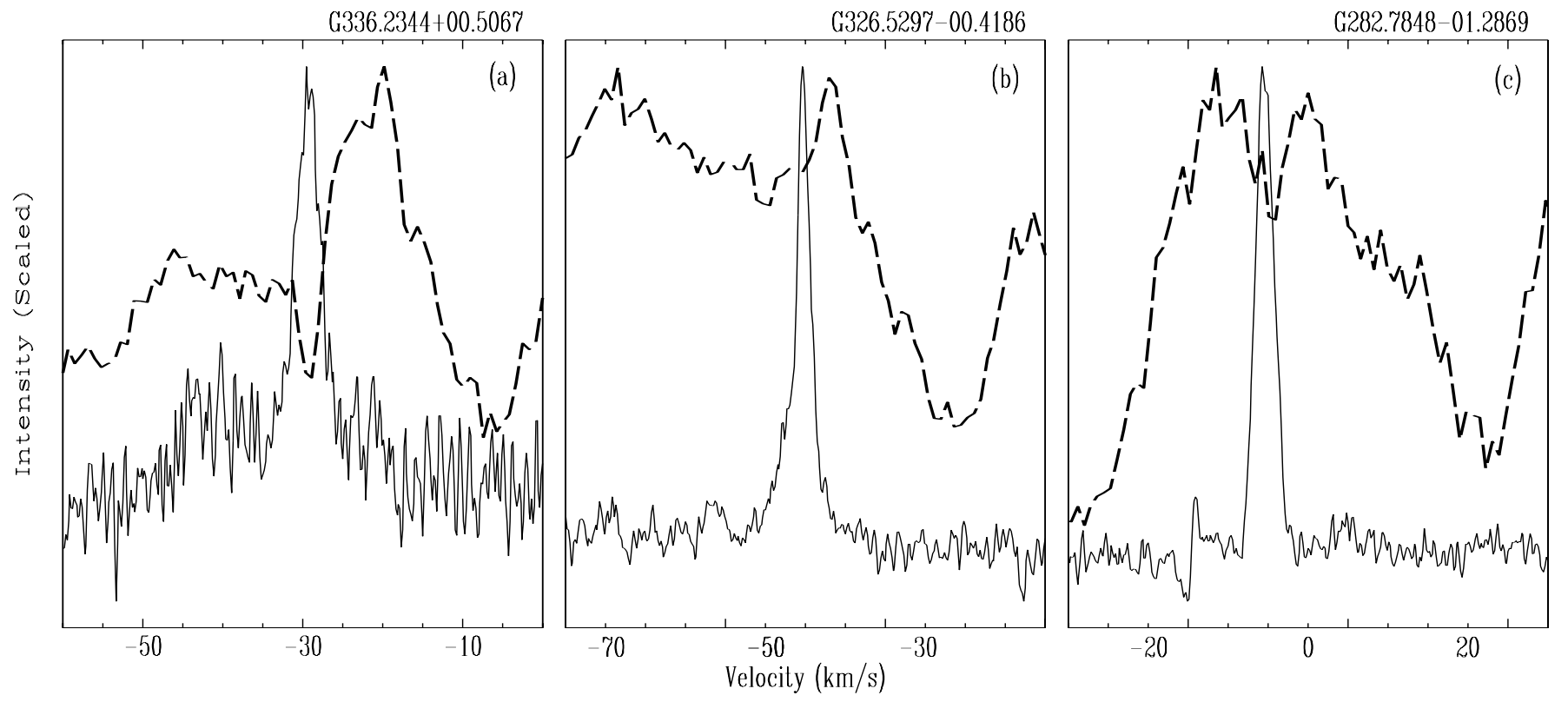

Figure 2. Representative spectra showing the three possible solutions obtained using the H I self-absorption method. The solid line represents the ${ }^{13} \mathrm{CO}$ spectra overlaid with the $\mathrm{H}$ I $21 \mathrm{~cm}$ data (bold dashed line), with the $\mathrm{H}$ I data scaled to the peak of ${ }^{13} \mathrm{CO}$. Plot (a) shows a clear near distance solution with the ${ }^{13} \mathrm{CO}$ emission line coinciding with a $\mathrm{H}$ I absorption. Plot (b) represents a far distance object with no H I SA located at the ${ }^{13} \mathrm{CO}$ peak velocity, whereas plot (c) shows an example of an ambiguity, as overall the ${ }^{13} \mathrm{CO}$ peak lies in a $\mathrm{H}$ I trough, but within the trough is a spike, hence not providing any clear cut answer to the kinematic distance ambiguity.

distances for all objects and any line-of-sight components can be found in Table 1. Direct overlays of all the ${ }^{13} \mathrm{CO}$ 1-0 molecular line and $\mathrm{H}_{\mathrm{I}} 21 \mathrm{~cm}$ data for each of the RMS sources can be found in Appendix Figure A1

Many ${ }^{13} \mathrm{CO}$ spectra show more than one emission component from clouds at different distances along the line of sight, with an increase in the number of objects towards the Galactic centre.. The results also show that we can simultaneously solve the kinematic distance ambiguity to many of these other clouds along the line of sight. Of the other 114 component ${ }^{13} \mathrm{CO}$ peaks 31 overlap with $\mathrm{H}$ I SA, indicating near distance objects, and the lack of $\mathrm{H}$ I SA for 57 components imply far distance objects. Again 22 components were unable to provide a definite answer to the distance ambiguity and were designated a probable (best) solution. Four components were left undetermined.

The Hi SA technique is much easier to use in resolving multiple line-of-sight component kinematic distance ambiguities than using the $\mathrm{H} 110 \alpha$ recombination line and $\mathrm{H}_{2} \mathrm{CO}$ absorption technique (such as Sewilo et al. 2004). When using the RRL technique it is not always possible to determine which $\mathrm{H}_{2} \mathrm{CO}$ lines are being absorbed against which $\mathrm{H}$ II region if there are multiple $\mathrm{H}$ II regions within the beam. For example, if multiple radio recombination lines are detected towards a source, and only one component with a distinct absorption, it is not possible to determine which $\mathrm{H}$ II region is responsible for the absorption, and hence resolve the distance ambiguity to these sources. Using the $\mathrm{H}$ I SA technique a direct comparison of both data sets is easily achieved without any similar problems, even if multiple clouds may still be located within the beam.

\subsection{Limitations due to complex line profiles}

Approximately $16 \%$ of objects are either self-absorbed or have a double-peaked profile at ${ }^{13} \mathrm{CO}$ (eg. G299.5265+00.1478). Structure in the ${ }^{13} \mathrm{CO}$ line makes it more difficult to match features in the $\mathrm{H}$ I spectrum. Self absorption, while uncommon in ${ }^{13} \mathrm{CO}$, can reduce the overall line strength making it weaker, whilst also affecting matching up potential dips with the line profile when overlaid onto HI. This may push the result to either a near or far distance depending on the peak of the stronger line. The overall line profile was considered when overlaid onto the $\mathrm{HI}$ data where cases with suspected ${ }^{13} \mathrm{CO}$ self absorption occurs and noted in the results table.

A similar strategy to the self-absorbed lines was used when ${ }^{13} \mathrm{CO}$ emission at the off position occurred at the velocity of the ${ }^{13} \mathrm{CO}$ emission. These objects in the off beam are due to high Galactic source crowding towards the inner plane of the Galaxy (eg. G338.2459-00.6115). Although great care was taken to try and reduce this effect whilst observing, some off beam objects still remain. The overall line profile again was considered before any decisions were made.

A few ${ }^{13} \mathrm{CO}$ lines had saturated flat tops, or structured peak profile such as G337.0642-01.1723, providing a unique problem. If we were to consider the line profile as a whole, one could say it should be at the the far distance or ambiguous. However, just considering the spiked edge, this lies well within a H I dip, and hence would be located at the near distance. For objects such as this the distance remains ambiguous. Similarly, ${ }^{13} \mathrm{CO}$ peaks lying halfway down a side of a H I dip (e.g. G311.9799-00.9527) again provide similar problems. The sources that remain ambiguous are noted in Appendix Table A1.

Noisy H I profiles are sometimes seen towards the upper 


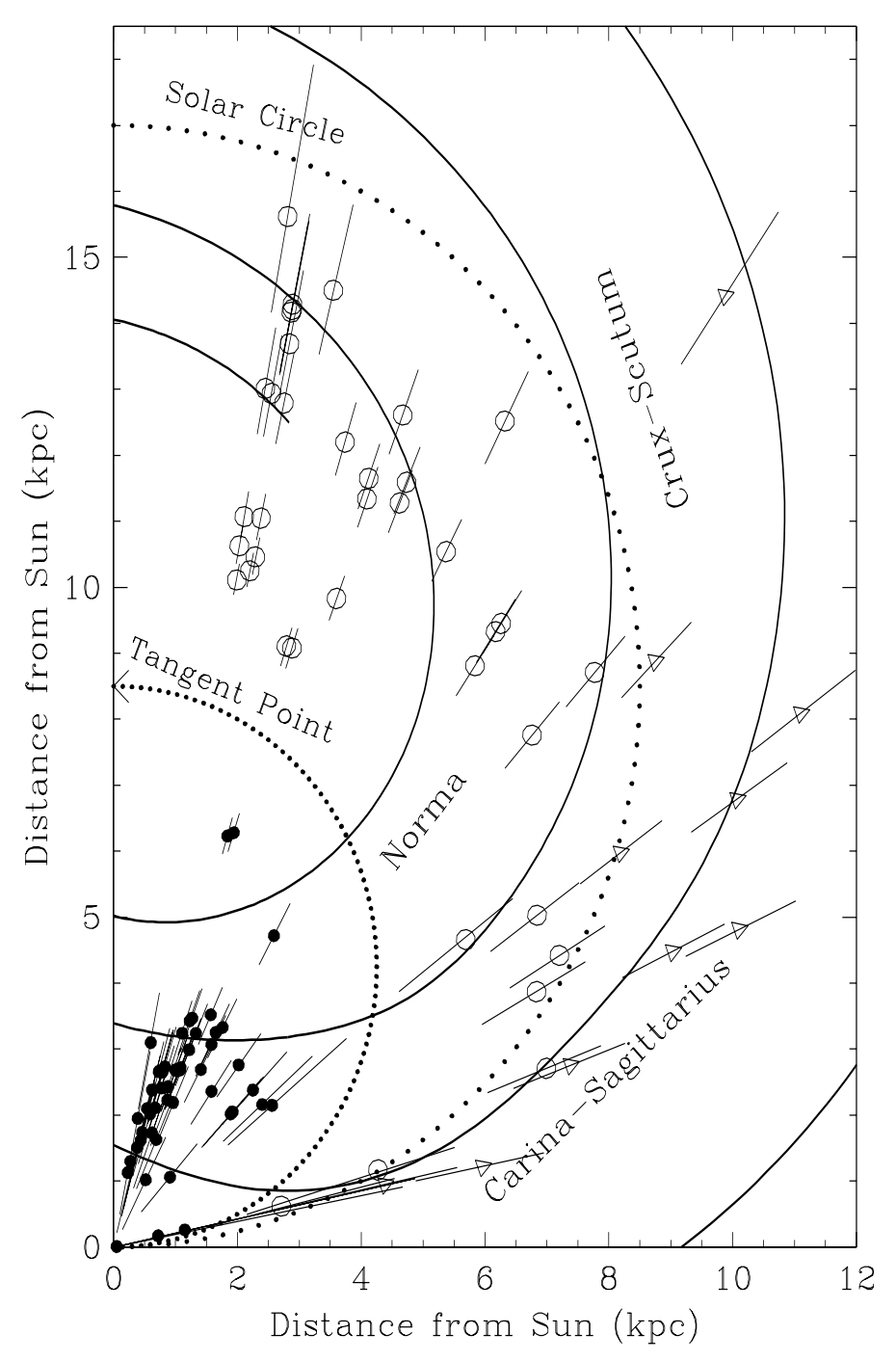

Figure 3. Galactic plot showing the distance ambiguity problem after comparison to the $\mathrm{H}$ I data. Filled circles in the inner (tangent) circle represent near distances, whereas the open circles represent far distances within in the outer (solar) circle. Open triangles represent the 10 sources which were studied with no distance ambiguity, and a typical peculiar velocity error of $\pm 10 \mathrm{~km}$ $\mathrm{s}^{-1}$ is indicated on each point. The updated spiral arm model of Tavlor \& Cordes (1993) by Cordes \& Lazio (2004) is indicated by solid lines.

or lower edges $\left(b> \pm 1.3^{\circ}\right)$ of a spectral cube due to more sparsely sampled and weaker Hi data at these positions. This reduces the quality and resolution of the $\mathrm{H}$ I data such as with object G340.2637-01.3236, and also complicates the comparison of the two spectra.

One interesting question is how big an absorption in $\mathrm{HI}$ is required to classify the object in question to be at a near or far distance. For objects which do not have other features affecting the classification of a near or far distance, a dip of $\sim 15-20 \%$ of the surrounding continuum intensity was required (shown by Flvnn et al. (2004)) to classify a near distance object. Components that had less H I absorption remain ambiguous, however, were designated a proba- ble near or far solution as indicated by features explained in Appendix Table A1.

\subsection{Further testing of the $\mathrm{H}_{\text {I }} \mathrm{SA}$ method}

The likelihood of detecting H I SA favours the geometry in which a molecular cloud lies at the near kinematic distance. Since H I exists everywhere throughout the Galaxy, it emits at essentially every allowed velocity. For clouds at the near kinematic distance, there is an ample amount of $\mathrm{H}$ i at the far distance and at the same velocity against which a cold molecular foreground cloud can be seen. If the same cloud were placed at the far distance, however, there should be little or no background $\mathrm{HI}$, and the SA feature would be absent. In addition, any weak SA for far clouds would be filled in by emission from warm clouds in the foreground. Note object G291.1881-00.2729 component 1, which is physically solved to be at the far distance, yet shows very slight H i SA features within its spectrum for example.

H I self-absorption may occur for clouds at the far kinematic distance, from noncircular velocities due to random cloud motions or streaming motions due to spiral density waves. This could change a distant cloud's velocity enough to place it in front of a significant column of gas which is at the same LSR velocity, but located behind the cloud at the far kinematic distance.

This led us to testing a further sample of 10 objects which are geometrically solved to be located outside the solar circle. Hence, they have no distance ambiguity associated with them due to a breakdown of the solutions to the kinematic distance ambiguity. These should contain no H I SA at the corresponding ${ }^{13} \mathrm{CO}$ velocity. Direct overlays of all the ${ }^{13} \mathrm{CO} 1-0$ molecular line and $\mathrm{H}$ I $21 \mathrm{~cm}$ data for each of the geometrically solved MYSO candidates can be found in Appendix Figure A2 For 7 of these objects there is no Hi SA at the ${ }^{13} \mathrm{CO}$ velocity indicating a clear far distance solution as expected. However, for the other 3 objects, G281.596100.1362, G282.2992-00.7763 and G306.1260-01.1371 there is a slight dip in each of the $\mathrm{H}$ I spectra at the ${ }^{13} \mathrm{CO}$ velocity position, indicating $\mathrm{H}$ I being located at that velocity, behind the object in question. This may be explained by looking at their location within the Galaxy. G281.5961-00.1362 and G282.2992-00.7763 are both tangential along the line-ofsight to the Carina-Sagittarius arm, with the arm extending far behind them. This could provide the streaming motions required to put the $\mathrm{H}$ I column of gas at the same LSR velocity but located behind the cloud at the far kinematic distance. G306.1260-01.1371 (and G282.2992-00.7763) however, is located on the edge of the solar circle between two spiral arms, and hence is only 'just' solved geometrically and may have peculiar velocities associated with it that could allow the solution to pass over the solar circle (i.e. a $\mathrm{v}_{L S R}<0$ ), and hence have a kinematic distance ambiguity. Ignoring those objects for which a peculiar velocity of $10 \mathrm{~km} \mathrm{~s}^{-1}$ would allow the solution to pass over the solar circle and have a kinematic distance ambiguity, the method can be seen to be successful in $85 \%$ (6 from 7 ) of cases.

A further confidence test was performed on the entire data set by randomly shifting the ${ }^{13} \mathrm{CO}$ spectrum along in velocity and re-performing the $\mathrm{H}$ I $\mathrm{SA}$ technique on the shifted data. This was performed in order to check the random chance of a $\mathrm{H}$ I dip lining up with a ${ }^{13} \mathrm{CO}$ component. 
The ${ }^{13} \mathrm{CO}$ spectra were moved by both $\pm 10 \mathrm{~km} \mathrm{~s}^{-1}$ and replotted over the $\mathrm{H}$ i line data. Approximately $80 \%$ of the resultant matches implied a far distance i.e. no $\mathrm{H}$ I $\mathrm{SA}$, with only $10 \%$ of the ${ }^{13} \mathrm{CO}$ components randomly coinciding with a $\mathrm{H}$ I dip implying a near distance. A further $10 \%$ of components were unable to be determined, again due to features mentioned previously. This implys an $80 \%$ success rate in determining the correct designation of whether an object is at the near or far distance, although the numbers tested mean this rate is uncertain by $14 \%$.

\subsection{Tracing Galactic structure}

As a check when dealing with distance ambiguities related to massive star formation, it is natural to ask whether the RMS survey sources studied follow any Galactic spiral arm structure, as it is well known that young massive stars are good tracers of Galactic spiral structure in galaxies (Georgelin \& Georgelin 1976).

Figures 3 and 4 help in trying to answer this question. Figure [3] shows the projected location in the Galactic plane of all our sources after solving the distance ambiguity problem. In this figure only the 4th Galactic quadrant is shown, with the Sun's location at the origin, and the Galactic centre at $(0,8.5)$ kpc. Figure 3 shows only those points for which the distance ambiguity can be reasonably resolved. Distance errors relating to peculiar velocities of $\pm 10 \mathrm{~km} \mathrm{~s}^{-1}$ are also shown. Although deviations from circular motion of up to $\pm 15 \mathrm{~km} \mathrm{~s}^{-1}$ can easily be found within the Galaxy Russeil 2003), these are generally more localised along the spiral arms.

Crowding towards the inner Galaxy can clearly be seen as expected, especially with the near distance sources, however no sharp or well-defined spiral structure can be easily seen. Objects at the far distance tend to lie on or near spiral arms, however, this is not always the case. Nearby objects towards the inner Galaxy tend to be much worse, and when taking into account potential peculiar velocities, some could lie on either of two Galactic arms. Also those inner Galactic sources with $l>340^{\circ}$ give more unreliable distances due to radial velocities being dominated by peculiar motions rather circular velocities.

The longitude-velocity $(l-v)$ plot shown in Figure 4 is an attempt to reveal the spiral structure using only observed quantities. The positions of the sources and components are independent of any Galactic rotation model, hence show no ambiguity. The spiral arm centroids are again plotted from the updated model of Cordes \& Lazid (2004). The obvious location crowding can again be seen towards the inner Galaxy as many of the Galactic arms overlap in the velocity domain. The tangent points of the outer two Galactic arms can also be picked out. Although no major Galactic structure can be ascertained from such a small sample, one interesting feature clearly seen is the $3 \mathrm{kpc}$ expanding arm. These sources are located towards the inner Galaxy by the Norma arm of the Galaxy running down towards a Galactic longitude of $340^{\circ}$ (indicated by a short line), matching the $3 \mathrm{kpc}$ expanding arm from the large-scale CO surveys of Dame. Hartmann \& Thaddeus (2001). This could also account for the group of sources in Figure 3 located inside the Norma arm towards the Galactic centre.

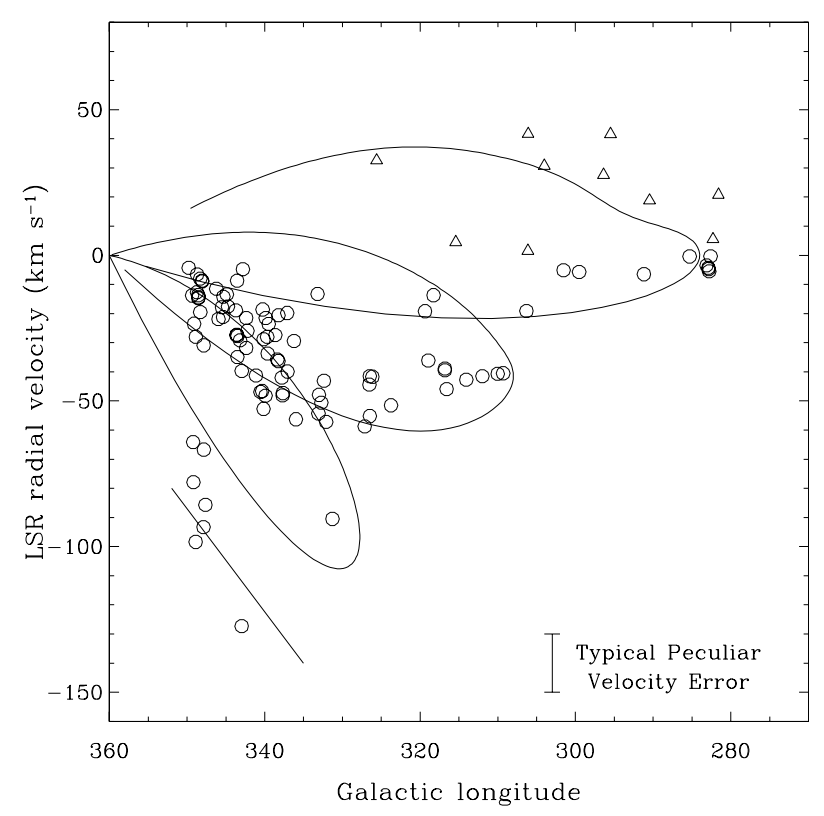

Figure 4. Longitude-velocity plot showing the distribution of our sources (open circles) in velocity space (i.e. no ambiguity). Open triangles represent the 10 sources which were studied with no distance ambiguity. Over-plotted are the Galactic arms from the model of Cordes \& Lazio (2004). The three Galactic arms shown are from upper to lower: Carina-Sagittarius, Crux-Scutum and Norma arm. The short straight line represents the $3 \mathrm{kpc}$ expanding arm. A typical peculiar velocity error $\pm 10 \mathrm{~km} \mathrm{~s}^{-1}$ is shown, allowing many of the sources to be positioned on two Galactic arms towards the inner Galaxy.

\section{CONCLUSIONS}

We used ${ }^{13} \mathrm{CO} 1-0$ and $\mathrm{N}_{2} \mathrm{H}^{+}$observations combined with SGPS H I data to resolve the kinematic distance ambiguity problem towards 94 RMS survey candidate MYSOs. The main motivation for this was to obtain accurate distances to potential MYSOs within the inner Galaxy, whilst also testing whether the H I SA technique is valid on our initial sample of sources. All sources studied had ${ }^{13} \mathrm{CO} 1-0$ spectral line or $\mathrm{N}_{2} \mathrm{H}^{+}$detections, with just over $55 \%$ containing multiple velocity components along the line-of-sight in ${ }^{13} \mathrm{CO}$.

The majority (66) of the 94 RMS survey MYSO candidates with a distance ambiguity were resolved using this method, with a further 24 objects having features that were unable to provide a definite answer to the distance ambiguity. Other line-of-sight velocity components present in the spectra were also solved simultaneously by this method.

The sources with resolved distance ambiguities were plotted on Galactic position-position and $l-v$ plots to determine if spiral arm patterns could be clearly discerned. No obvious Galactic pattern could be seen, likely due to such a small sample size. Once typical data scatter introduced by peculiar velocities is allowed for, the source distance scatter can be the separation of the spiral arms themselves. The positions of the sources in the velocity domain provide a slightly better picture with the majority lying near loci of spiral arms, including a small group of sources following the $3 \mathrm{kpc}$ expanding arm.

Obtaining accurate distances to our RMS survey MYSO candidates is crucial in helping to determine the physi- 
cal properties (e.g. luminosities) of these potential massive young stellar objects and the surrounding star formation regions. Whilst this is a relatively unstudied quadrant of the Galaxy, with respect to distance ambiguities, the completion of the SGPS now gives us access to $\mathrm{H}$ I data in the entire southern hemisphere, allowing now for a much more thorough test of the H I SA technique. With the majority of the IGPS H I data being readily available, this provides a wealth of information, ripe for large scale (kinematic) surveys such as our own RMS survey.

\section{ACKNOWLEDGEMENTS}

We would like to thank Naomi McClure-Griffiths for her time and patience in providing all the $\mathrm{H}$ I data cubes which we required. We would also like to thank the anonymous referee whose comments provided a useful change of the structure and reasoning of the paper.

\section{REFERENCES}

Araya E., Hofner P., Churchwell E., Kurtz S., 2002, ApJS, 138,63

Araya E., Hofner P., Churchwell E., Kurtz S., 2001, AAS, $1991,2402 \mathrm{~A}$

Burton W. B., Liszt H. S., Baker P. L., 1978, ApJ, 219, L67

Brand J., Blitz L., 1993, A\&A, 275, 67

Cordes J. M., Lazio T. J. W., 2004, ASPC, 317, 211

Dame T. M., Hartmann D., Thaddeus P., 2001, ApJ, 547, 792

Downes D., Wilson T. L., Bieging J., Wink J., 1980, A\&AS, 40, 379

English J., et al., 1998, PASA, 15, 56

Flynn E. S., Jackson J. M., Simon R., Shah R. Y., Bania T., 2004, ASP Conference Series, 317, 44

Georgelin Y. M., Georgelin Y. P., 1976, A\&A, 49, 57

Henning T., Friedemann C., Guertler J., Dorschner J., 1984, Astron. Nachr. 305, 67

Hoare M. G., Lumsden S. L., Oudmaijer R. D., Busfield A. L., King T. L., Moore T. L. J., 2004, ASP Conf. Series, 317,156

Jackson J. M., Bania T. M., Simon R., Kolpak M., Clemens

D. P., Heyer M., 2002, ApJ, 566, 81

Kolpak M. A., Jackson J. M., Bania T. M., Clemens D. P., Dickey J. M., 2003, ApJ, 582, 756

Kuchar T., A. Bania T. M., 1993, ApJ, 414, 664

Liszt H. S., Burton W. B., Bania T. M., 1981, ApJ, 246,74

Lumsden S. L., Hoare M. G., Oudmaijer R. D., Richards D., 2002, MNRAS, 336, 621

McClure-Griffiths N., Dickey J. M., Gaensler B. M., Green A. J., Haynes R. F., Wieringa M. H., 2001, PASA, 18, 84 McClure-Griffiths N., Green A. J., Dickey J. M., Gaensler B. M., Haynes R. F., Wieringa M. H., 2001, ApJ, 551, 394.

Paladini R., Davies R. D., DeZotti G., 2004, MNRAS, 347

237

Russeil D., 2003, A\&A, 397, 133

Sewilo M., Watson C., Araya E., Churchwell E., Hofner P., Kurtz S., 2004, ApJS, 154, 553
Sridharan T. K., Beuther H., Schilke P., Menten K. M., Wyrowski F., 2002, ApJ, 566, 931

Taylor J. H., Cordes J. M., 1993, ApJ, 411, 674

Taylor A. R., Stil J. M., Dickey J. M., McClure-Griffiths N. M., Martin P. G., Rothwell T., Lockman F. J., 2002, ASP Conf. Series, 276, 68

Wink J. E., Altenhoff W. J., Mezger P. G., 1982, A\&A, 108,227

Wouterloot J. G. A., Brand J., 1989, A\&AS, 80, 149

Wu Y., Wu J., Wang J., 2001, A\&A, 380, 665

\section{APPENDIX A: RESULTS}

We plot direct overlays of the ${ }^{13} \mathrm{CO} 1-0$ line and $\mathrm{H}$ I data for all objects with a distance ambiguity in Figure A1

Overlays of all the ${ }^{13} \mathrm{CO} 1-0$ molecular line and $\mathrm{H}$ i $21 \mathrm{~cm}$ data for each of the geometrically solved MYSO candidates can be found in Figure $\mathrm{A} 2$

Tabulated results of all calculated parameters for all objects with a distance ambiguity can be found in Table A1. All calculated parameters for the 10 objects which are geometrically solved to be at the far distance can be found in Table A2. 

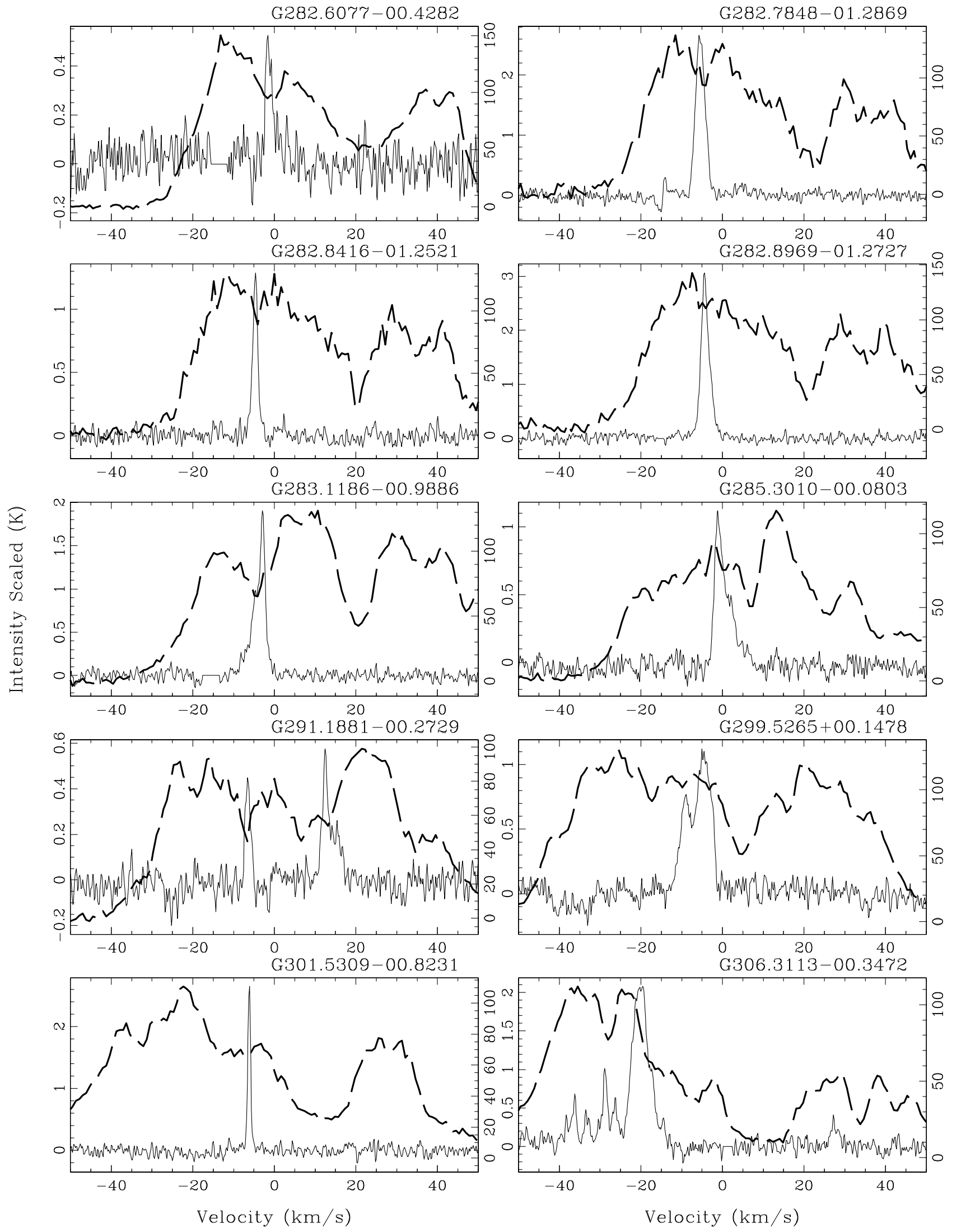

Figure A1. Plots showing the direct overlays of the ${ }^{13} \mathrm{CO}$ 1-0 line and $\mathrm{H}$ I data. The bold dashed line spectra represents the $\mathrm{H}$ I through the Galaxy, whereas the solid line is the ${ }^{13} \mathrm{CO}$ spectra of the $M S X$ object in question with the peak scaled to that of $\mathrm{H}$ I. An asterisk after the MSX name represents a $\mathrm{N}_{2} \mathrm{H}^{+}$line being used instead of ${ }^{13} \mathrm{CO}$ line. The left and right hand axes represent ${ }^{13} \mathrm{CO}$ and $\mathrm{HI}$ intensities respectively. 


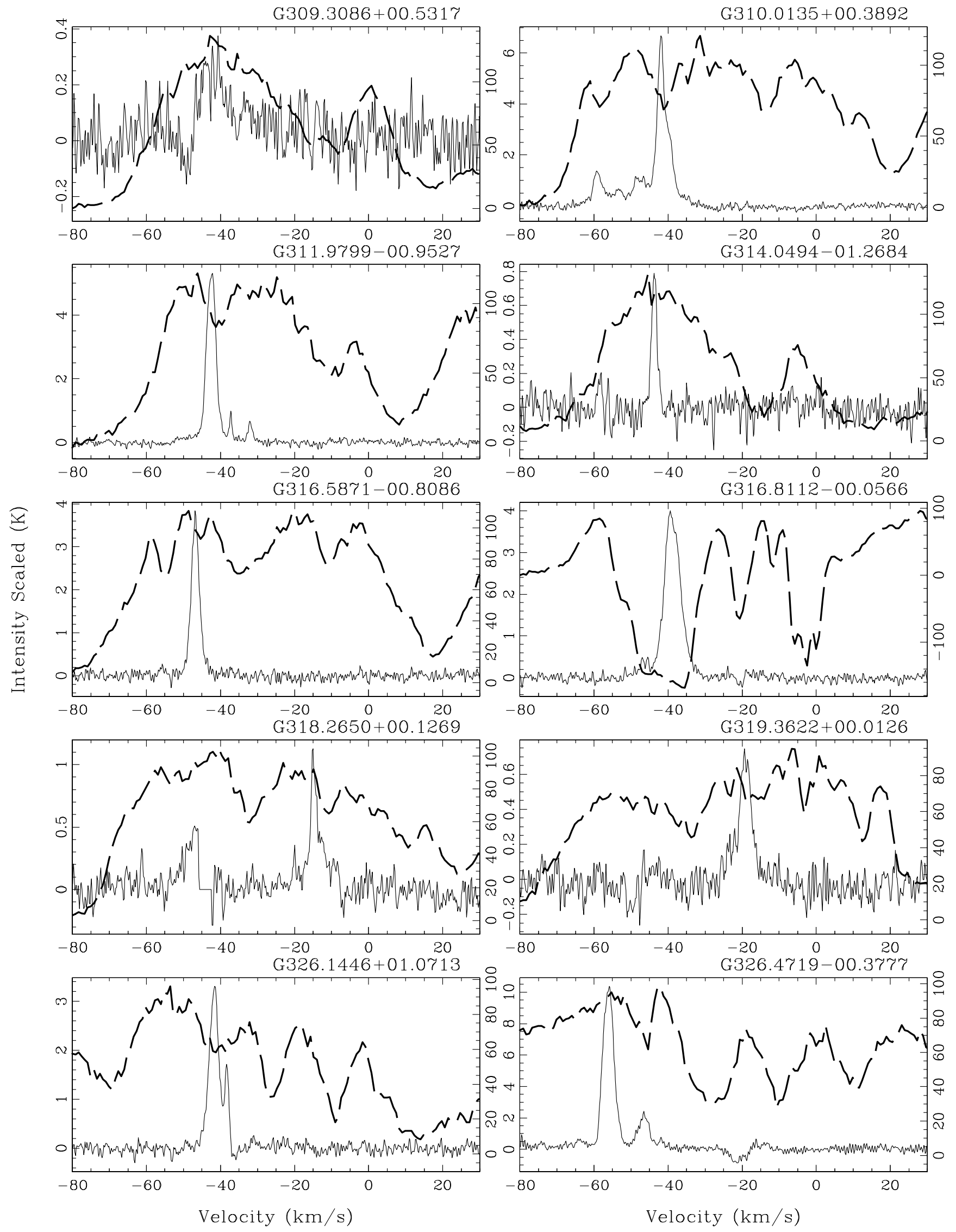

Figure A1 - continued 


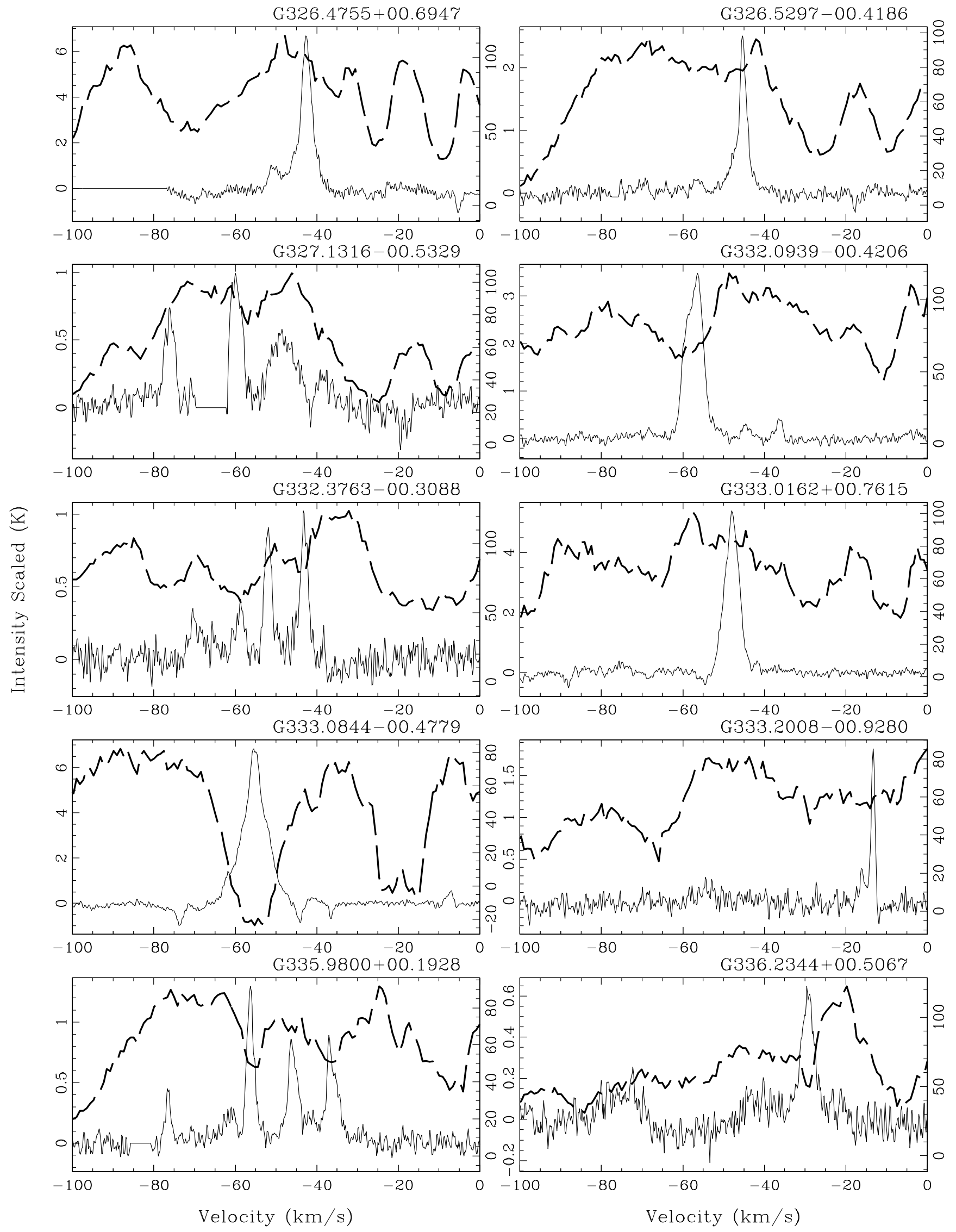

Figure A1 - continued 

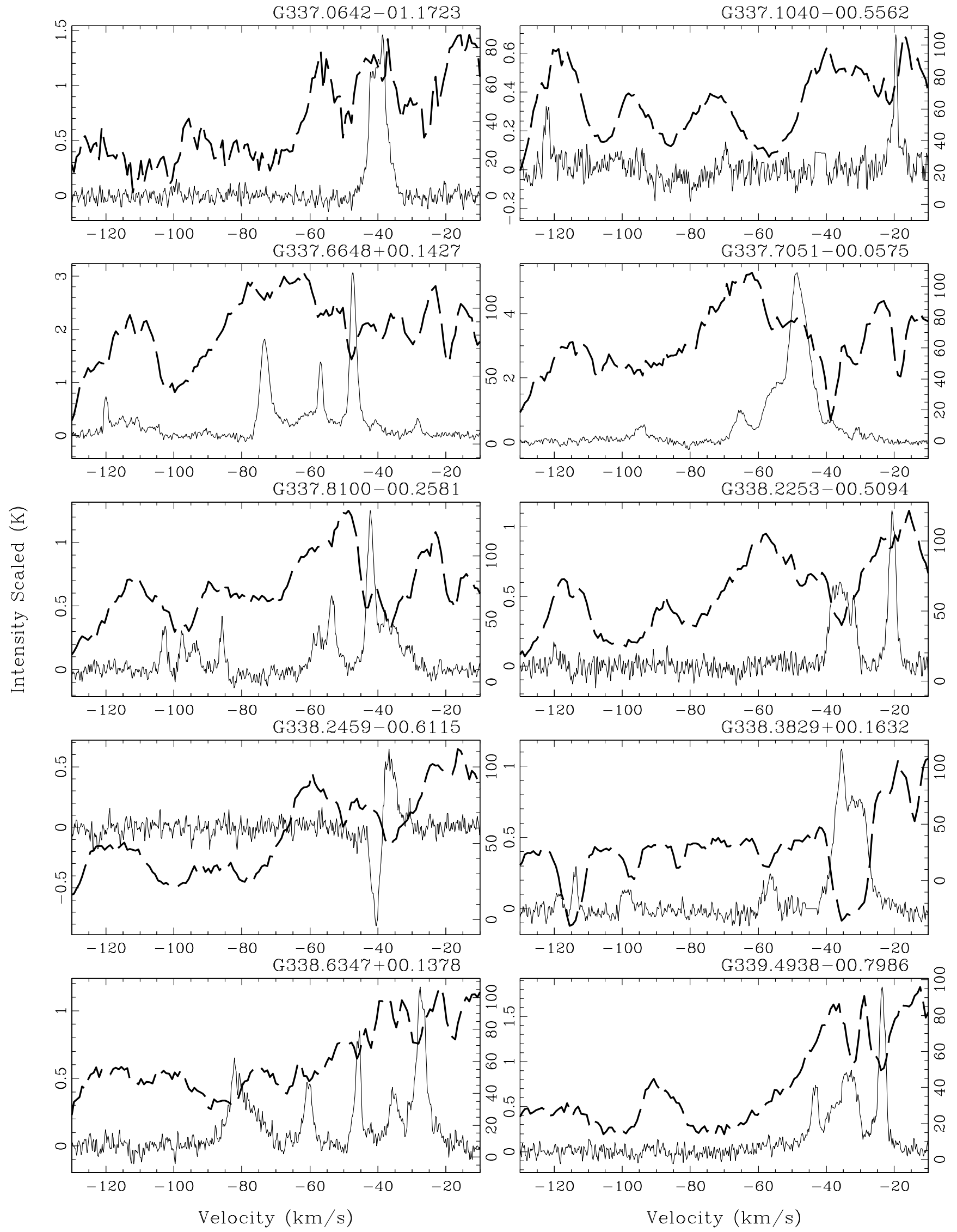

Figure A1 - continued 

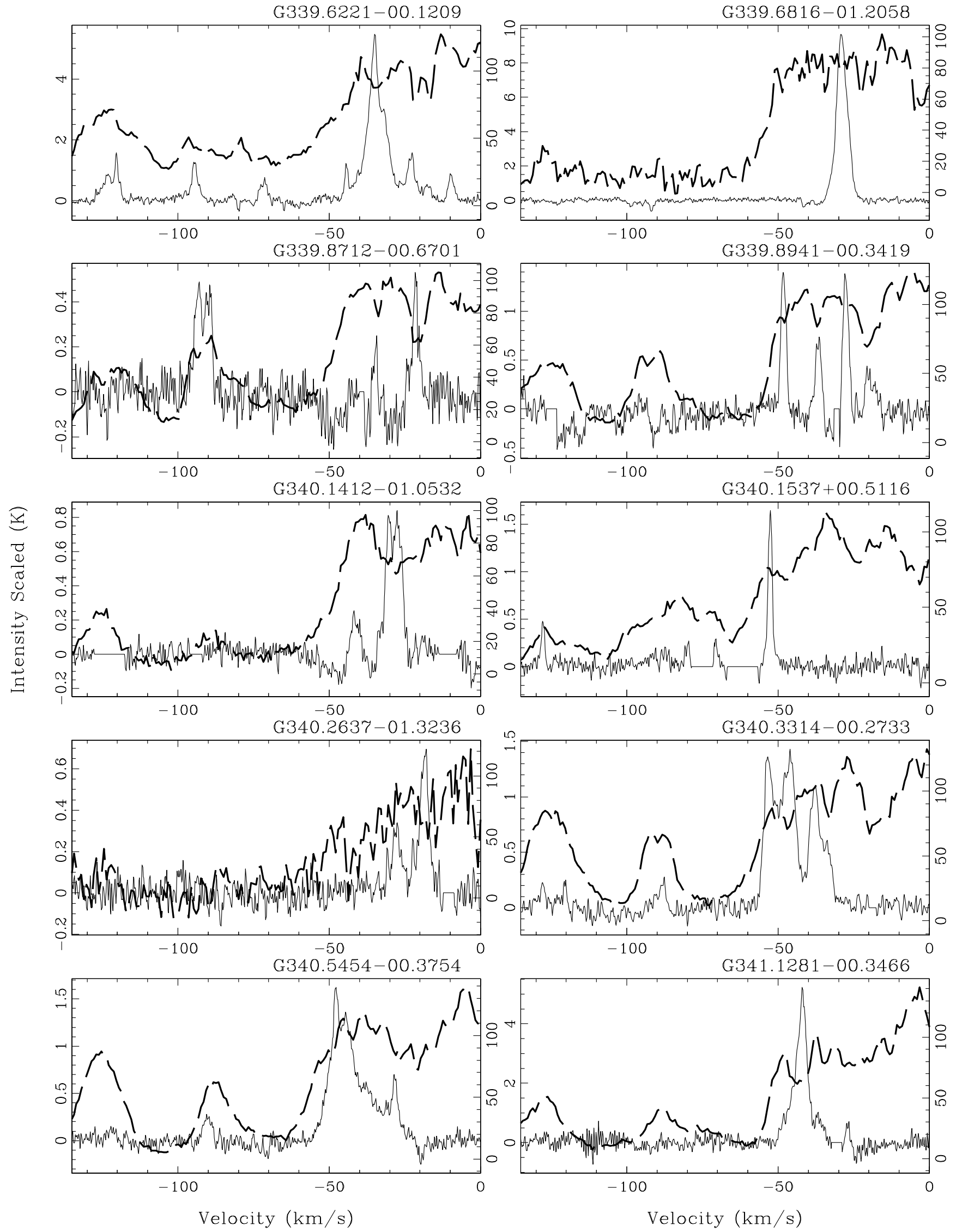

Figure A1 - continued 
Resolving the Kinematic Distance Ambiguity of Southern Massive Young Stellar Object Candidates

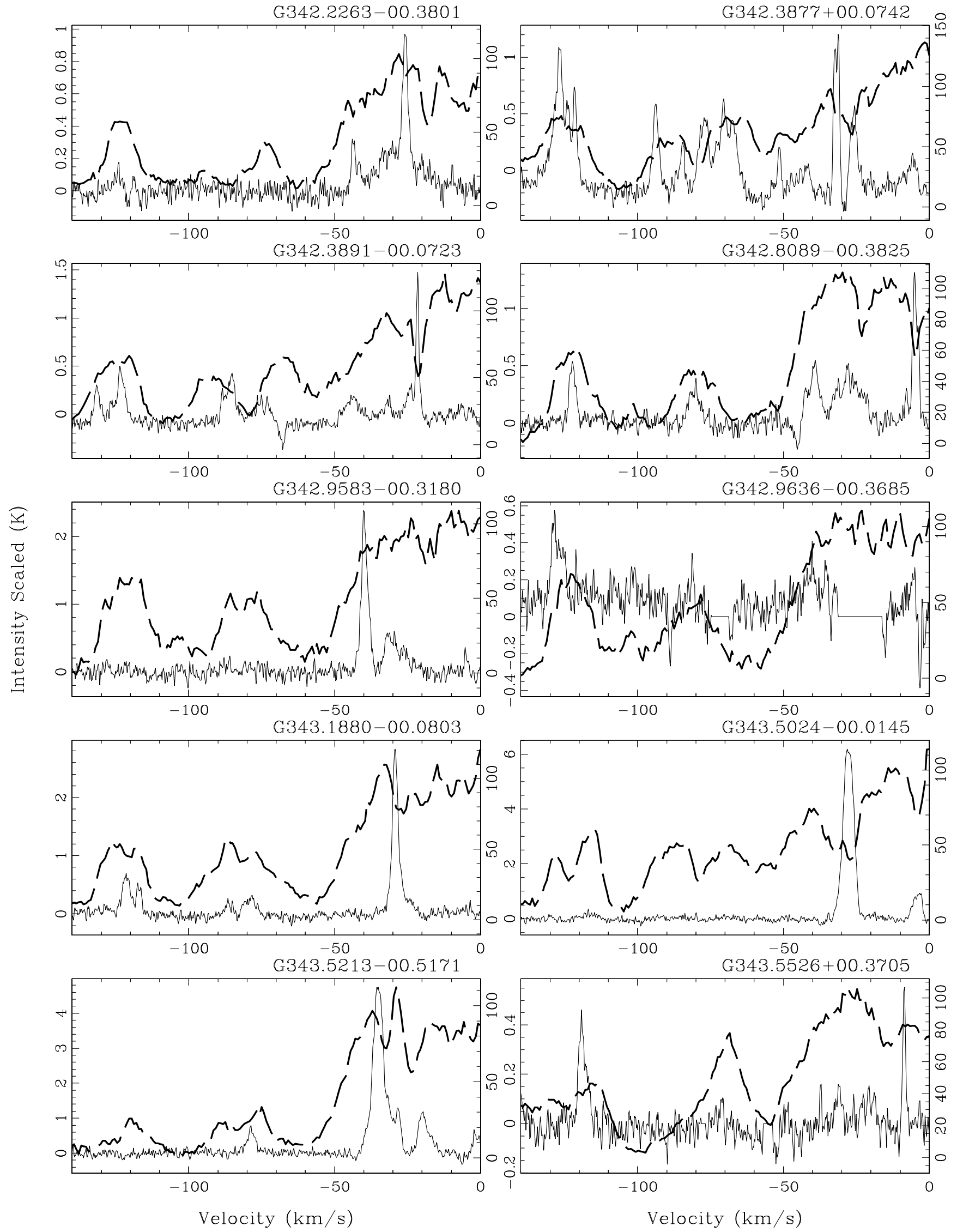

Figure A1 - continued 


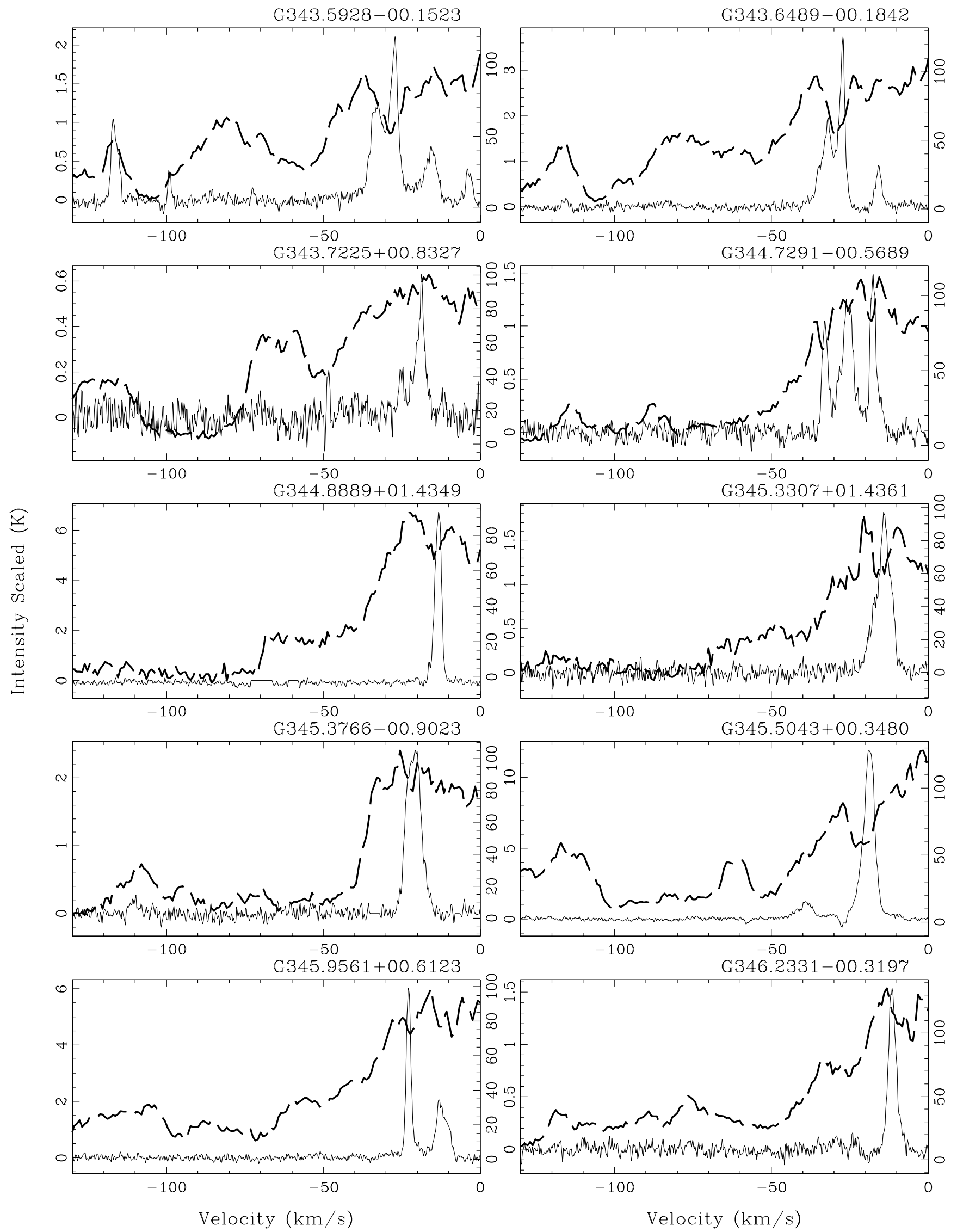

Figure A1 - continued 
Resolving the Kinematic Distance Ambiguity of Southern Massive Young Stellar Object Candidates
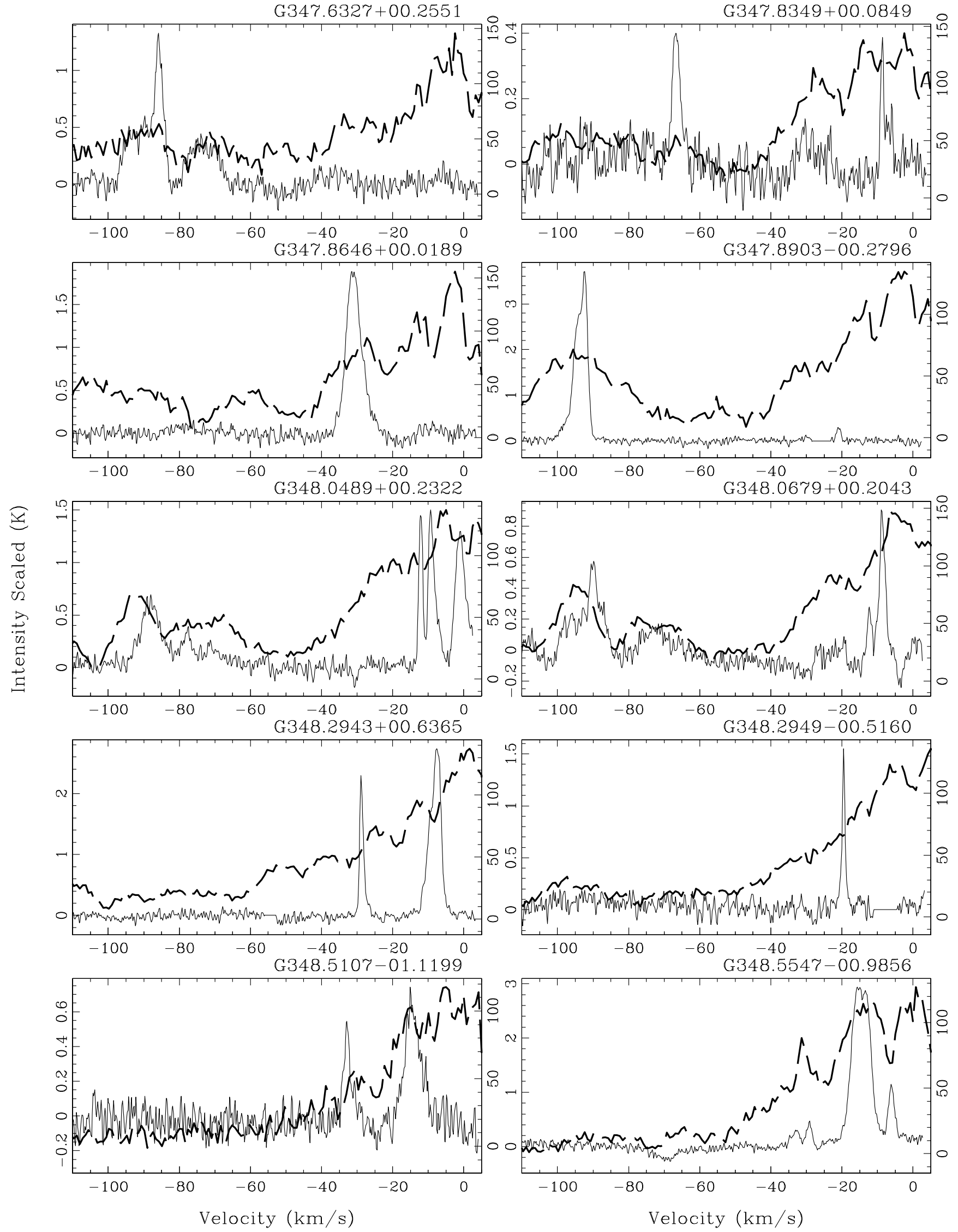

Figure A1 - continued 


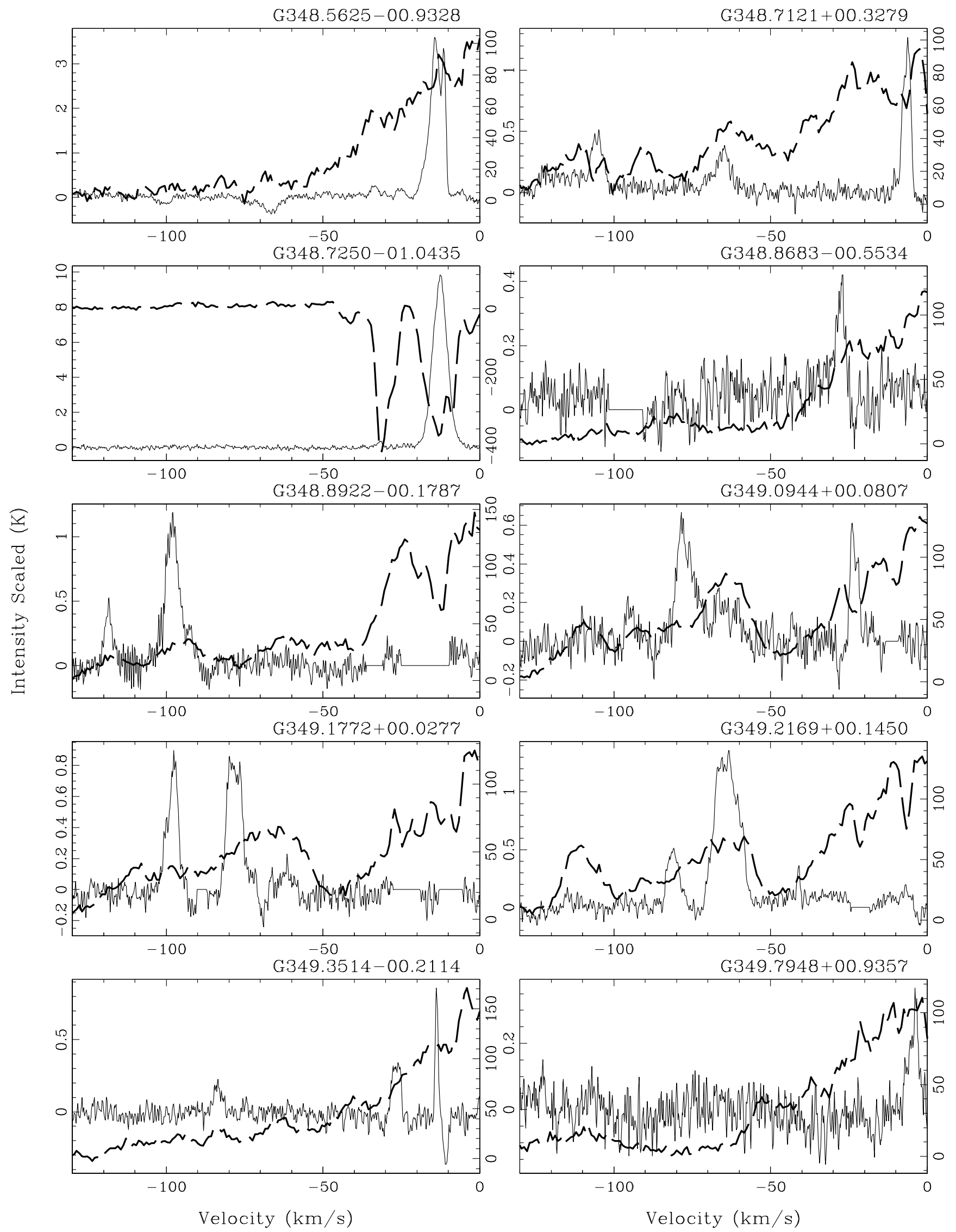

Figure A1 - continued 

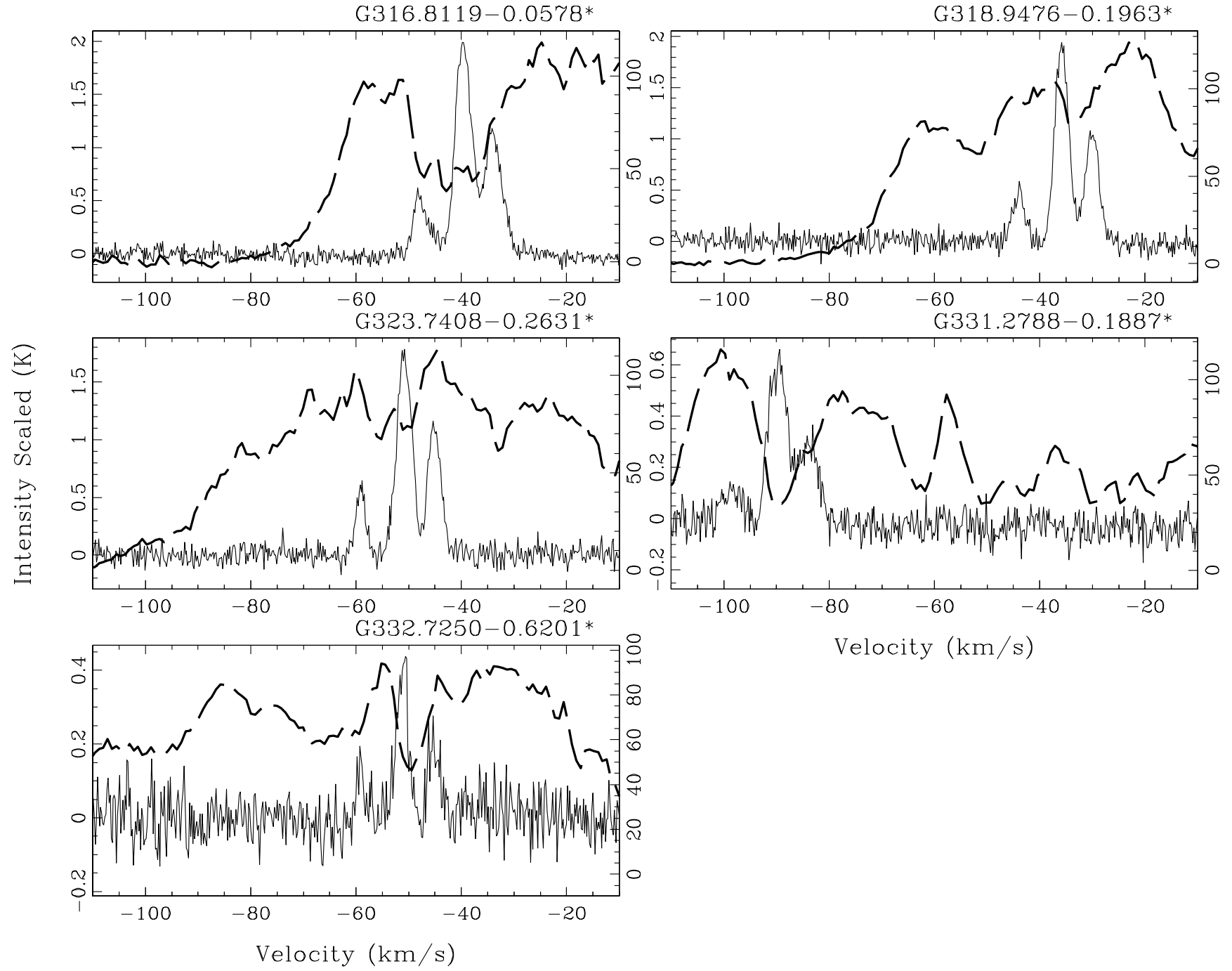

Velocity $(\mathrm{km} / \mathrm{s})$ 

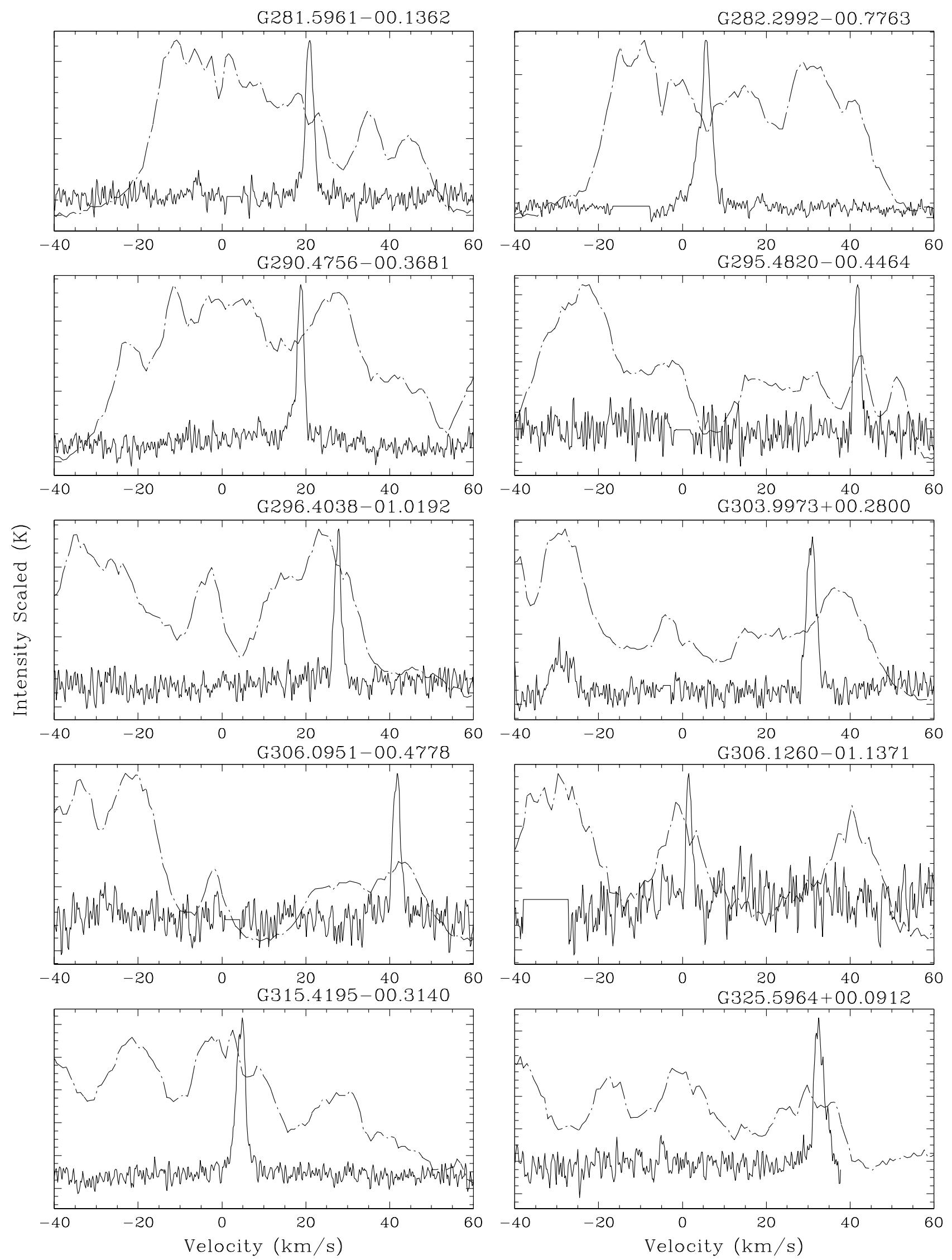

Figure A2. Plots showing the direct overlays of the ${ }^{13} \mathrm{CO} 1-0$ line and $\mathrm{H}$ I data on objects that are geometrically solved to be outside the solar circle, and hence have no distance ambiguity associated with them. The dashed line spectra represents the H I through the Galaxy, whereas the solid line is the ${ }^{13} \mathrm{CO}$ spectra of the $M S X$ object in question. 
Table A1. ${ }^{13} \mathrm{CO}$ 1-0 Kinematic parameters of $M S X$ sources with a distance ambiguity

\begin{tabular}{|c|c|c|c|c|c|c|c|c|}
\hline Source Name & & $\begin{array}{c}\mathrm{V}_{L S R} \\
\left(\mathrm{~km} \mathrm{~s}^{-1}\right)\end{array}$ & $\begin{array}{l}\mathrm{D}_{\text {near }} \\
(\mathrm{kpc})\end{array}$ & $\begin{array}{l}\mathrm{D}_{\text {far }} \\
(\mathrm{kpc})\end{array}$ & $\begin{array}{c}\mathrm{z} \\
(\mathrm{pc})\end{array}$ & $\begin{array}{c}\mathrm{R}_{g c} \\
(\mathrm{kpc})\end{array}$ & $\begin{array}{l}\text { Adopted } \\
\text { Distance }\end{array}$ & Notes \\
\hline G282.6077-00.4282 & & -0.3 & $0.1(1.4)$ & 3.6 & -0.7 & 8.5 & $\mathrm{~N}$ & \\
\hline G282.7848-01.2869 & & -5.5 & 1.8 & 1.9 & - & 8.3 & $?$ & ambiguous \\
\hline G282.8416-01.2521 & & -4.7 & $1.2(1.9)$ & 2.6 & -26.2 & 8.3 & $\mathrm{~N}$ & \\
\hline G282.8969-01.2727 & & -4.3 & 1.0 & $2.8(1.8)$ & -62.2 & 8.3 & $\mathrm{~F}$ & \\
\hline G283.1186-00.9886 & & -3.5 & $0.7(1.6)$ & 3.1 & -12.1 & 8.4 & $\mathrm{~N} ?$ & \\
\hline G285.3010-00.0803 & & -0.4 & 0.1 & $4.4(1.2)$ & -6.2 & 8.5 & $\mathrm{~F}$ & \\
\hline \multirow{2}{*}{ G291.1881-00.2729 } & 1 & 13.0 & - & $7.5(0.9)$ & -35.7 & 9.1 & - & $\mathrm{n} / \mathrm{a}$ \\
\hline & 2 & -6.5 & $0.8(1.2)$ & 5.3 & -3.8 & 8.2 & $\mathrm{~N}$ & \\
\hline G299.5265+00.1478 & & -5.7 & 0.5 & $7.9(0.9)$ & 20.4 & 8.3 & $\mathrm{~F}$ & SA/off-beam? \\
\hline G301.5309-00.8231 & & -5.1 & 0.4 & $8.4(0.8)$ & -120.7 & 8.3 & $\mathrm{~F}$ & \\
\hline \multirow[t]{2}{*}{ G306.3113-00.3472 } & 1 & -19.1 & 1.6 & $8.5(0.8)$ & -51.5 & 7.7 & $\mathrm{~F}$ & \\
\hline & 2 & -27.2 & $2.3(0.9)$ & 7.8 & -13.9 & 7.4 & $\mathrm{~N}$ & \\
\hline G309.3086+00.5317 & & -40.5 & 3.4 & $7.4(0.9)$ & 68.7 & 6.9 & $\mathrm{~F}$ & off-beam \\
\hline \multirow[t]{3}{*}{ G310.0135+00.3892 } & 1 & -40.7 & $3.3(0.9)$ & 7.6 & 22.4 & 6.9 & $\mathrm{~N}$ & \\
\hline & 2 & -58.7 & - & - & - & 6.3 & - & Unphysical $\mathrm{V}_{L S R}$ \\
\hline & 3 & -47.5 & 4.2 & $6.7(1.1)$ & 45.5 & 6.6 & $\mathrm{~F}$ & \\
\hline \multirow[t]{3}{*}{ G311.9799-00.9527 } & 1 & -41.5 & $2.2(0.8)$ & 8.1 & -36.6 & 6.8 & $\mathrm{~N} ?$ & \\
\hline & 2 & -36.3 & 2.8 & $8.6(0.7)$ & -143.0 & 7.0 & $\mathrm{~F}$ & \\
\hline & 3 & -31.0 & $2.4(0.7)$ & 9.0 & -39.9 & 7.1 & $\mathrm{~N} ?$ & \\
\hline G314.0494-01.2684 & & -42.7 & $3.2(0.7)$ & $8.6(0.7)$ & - & 6.7 & $?$ & ambiguous \\
\hline G316.5871-00.8086 & & -45.9 & $3.3(0.7)$ & 9.1 & -46.6 & 6.5 & $\mathrm{~N} ?$ & \\
\hline G316.8112-00.0566 & & -38.9 & $2.8(0.6)$ & 9.6 & -2.8 & 6.8 & $\mathrm{~N}$ & \\
\hline \multirow[t]{2}{*}{ G318.2650+00.1269 } & 1 & -13.7 & 1.0 & $11.7(0.7)$ & 25.9 & 7.8 & $\mathrm{~F}$ & \\
\hline & 2 & -46.4 & 3.2 & $9.5(0.6)$ & 21.0 & 6.5 & $\mathrm{~F} ?$ & off-beam \\
\hline G319.3622+00.0126 & & -19.2 & $1.4(0.7)$ & 11.5 & 0.3 & 7.5 & $\mathrm{~N} ?$ & \\
\hline \multirow[t]{2}{*}{ G326.1446+01.0713 } & 1 & -41.7 & $2.8(0.5)$ & 11.3 & 52.4 & 6.3 & $\mathrm{~N} ?$ & SA/off-beam? \\
\hline & 2 & -38.3 & $2.6(0.6)$ & 11.5 & 48.6 & 6.5 & $\mathrm{~N} ?$ & \\
\hline \multirow[t]{2}{*}{ G326.4719-00.3777 } & 1 & -55.2 & 3.6 & $10.6(0.5)$ & -69.9 & 5.9 & $\mathrm{~F}$ & \\
\hline & 2 & -45.7 & $3.1(0.5)$ & 11.1 & -20.4 & 6.2 & $\mathrm{~N}$ & \\
\hline G326.4755+00.6947 & & -41.5 & 2.8 & $11.3(0.5)$ & 137.0 & 6.3 & $\mathrm{~F}$ & \\
\hline G326.5297-00.4186 & & -44.4 & 3.0 & $11.2(0.5)$ & -81.8 & 6.2 & $\mathrm{~F}$ & \\
\hline \multirow[t]{3}{*}{ G327.1316-00.5329 } & 1 & -47.4 & $3.2(0.5)$ & 11.1 & -29.8 & 6.1 & $\mathrm{~N} ?$ & strongest? \\
\hline & 2 & -58.7 & 3.8 & $10.5(0.5)$ & -97.7 & 5.7 & $\mathrm{~F}$ & off-beam \\
\hline & 3 & -75.2 & 4.7 & $9.6(0.5)$ & -89.3 & 5.2 & $\mathrm{~F}$ & \\
\hline \multirow[t]{2}{*}{ G332.0939-00.4206 } & 1 & -57.2 & $3.8(0.5)$ & 11.3 & -27.9 & 5.5 & $\mathrm{~N} ?$ & \\
\hline & 2 & -36.3 & 2.6 & $12.4(0.6)$ & -91.0 & 6.3 & $\mathrm{~F}$ & \\
\hline \multirow[t]{3}{*}{ G332.3763-00.3088 } & 1 & -43.1 & $3.0(0.5)$ & 12.0 & -16.2 & 6.0 & $\mathrm{~N}$ & \\
\hline & 2 & -51.9 & 3.5 & $11.6(0.5)$ & -62.5 & 5.6 & $\mathrm{~F}$ & \\
\hline & 3 & -58.8 & $3.9(0.5)$ & 11.2 & -21.0 & 5.4 & $\mathrm{~N}$ & \\
\hline G333.0162+00.7615 & & -47.9 & 3.3 & $11.8(0.5)$ & 156.8 & 5.7 & $\mathrm{~F}$ & \\
\hline \multirow[t]{2}{*}{ G333.0844-00.4779 } & 1 & -54.3 & $3.6(0.5)$ & 11.5 & -30.0 & 5.5 & $\mathrm{~N}$ & \\
\hline & 2 & -6.4 & 0.6 & $14.6(0.9)$ & -121.8 & 8.0 & $\mathrm{~F}$ & \\
\hline G333.2008-00.9280 & & -13.3 & 1.1 & $14.0(0.8)$ & -226.8 & 7.5 & $\mathrm{~F} ?$ & \\
\hline \multirow[t]{4}{*}{ G335.9800+00.1928 } & 1 & -56.3 & $3.9(0.5)$ & 11.7 & 13.1 & 5.2 & $\mathrm{~N}$ & \\
\hline & 2 & -45.9 & $3.3(0.5)$ & 12.2 & 11.1 & 5.6 & $\mathrm{~N} ?$ & \\
\hline & 3 & -36.4 & $2.8(0.6)$ & 12.7 & 9.4 & 6.0 & $\mathrm{~N}$ & \\
\hline & 4 & -76.5 & 4.8 & $10.8(0.4)$ & 36.3 & 4.6 & $\mathrm{~F}$ & \\
\hline G336.2344+00.5067 & & -29.4 & $2.4(0.6)$ & 13.2 & 21.2 & 6.4 & $\mathrm{~N}$ & \\
\hline G337.0642-01.1723 & & -39.9 & $3.1(0.6)$ & $12.6(0.6)$ & - & 5.8 & $?$ & ambiguous \\
\hline \multirow[t]{2}{*}{ G337.1040-00.5562 } & 1 & -19.8 & $1.8(0.8)$ & 13.9 & -17.5 & 6.9 & $\mathrm{~N} ?$ & \\
\hline & 2 & -122.0 & $6.7(0.4)$ & 9.0 & -65.0 & 3.5 & $\mathrm{~N}$ & \\
\hline \multirow[t]{5}{*}{ G337.6648+00.1427 } & 1 & -47.3 & $3.5(0.5)$ & 12.2 & 8.7 & 5.4 & $\mathrm{~N}$ & \\
\hline & 2 & -73.2 & $4.7(0.4)$ & 11.0 & 11.7 & 4.5 & $\mathrm{~N}$ & \\
\hline & 3 & -57.0 & 4.0 & $11.8(0.4)$ & 29.4 & 5.1 & $\mathrm{~F}$ & \\
\hline & 4 & -119.9 & 6.5 & $9.2(0.4)$ & 22.9 & 3.5 & $\mathrm{~F}$ & \\
\hline & 5 & -28.4 & $2.4(0.7)$ & 13.3 & 6.0 & 6.4 & $\mathrm{~N}$ & \\
\hline G337.7051-00.0575 & 1 & -48.1 & 3.5 & $12.2(0.5)$ & -12.2 & 5.4 & $\mathrm{~F}$ & structure \\
\hline
\end{tabular}


Table A1 - Continued.

\begin{tabular}{|c|c|c|c|c|c|c|c|c|}
\hline Source Name & & $\begin{array}{c}\mathrm{V}_{L S R} \\
\left(\mathrm{~km} \mathrm{~s}^{-1}\right)\end{array}$ & $\begin{array}{l}\mathrm{D}_{\text {near }} \\
(\mathrm{kpc})\end{array}$ & $\begin{array}{l}\mathrm{D}_{f a r} \\
(\mathrm{kpc})\end{array}$ & $\begin{array}{c}\mathrm{z} \\
(\mathrm{pc})\end{array}$ & $\begin{array}{c}\mathrm{R}_{g c} \\
(\mathrm{kpc})\end{array}$ & $\begin{array}{l}\text { Adopted } \\
\text { Distance }\end{array}$ & Notes \\
\hline \multirow{6}{*}{ G337.8100-00.2581 } & 2 & -56.4 & 3.9 & $11.8(0.4)$ & -11.8 & 5.1 & $\mathrm{~F}$ & \\
\hline & 3 & -65.0 & $4.3(0.4)$ & 11.4 & -4.3 & 4.8 & $\mathrm{~N} ?$ & \\
\hline & 1 & -42.0 & $3.2(0.5)$ & 12.5 & -14.4 & 5.7 & $\mathrm{~N}$ & \\
\hline & 2 & -54.5 & $3.9(0.5)$ & 11.9 & -17.6 & 5.1 & $\mathrm{~N} ?$ & \\
\hline & 3 & -85.9 & 5.2 & $10.5(0.3)$ & -47.3 & 4.2 & $\mathrm{~F}$ & \\
\hline & 4 & -102.9 & 5.8 & $9.9(0.3)$ & -44.6 & 3.8 & $\mathrm{~F}$ & \\
\hline \multirow{3}{*}{ G338.2253-00.5094 } & 5 & -95.4 & 5.6 & $10.2(0.3)$ & -45.9 & 4.0 & $\mathrm{~F}$ & SA/off-beam? \\
\hline & 1 & -35.6 & $2.9(0.6)$ & 12.9 & -25.8 & 5.9 & $\mathrm{~N}$ & strongest? \\
\hline & 2 & -20.6 & $1.9(0.8)$ & 13.9 & -16.9 & 6.8 & $\mathrm{~N}$ & \\
\hline G338.2459-00.6115 & & -36.3 & $2.9(0.6)$ & 12.9 & -31.0 & 5.9 & $\mathrm{~N}$ & off-beam \\
\hline \multirow[t]{4}{*}{ G338.3829+00.1632 } & 1 & -35.8 & $2.9(0.6)$ & 12.9 & 8.3 & 5.9 & $\mathrm{~N}$ & structure \\
\hline & 2 & -113.8 & $6.3(0.3)$ & 9.5 & 17.9 & 3.5 & $\mathrm{~N}$ & \\
\hline & 3 & -56.6 & $4.0(0.4)$ & 11.8 & 11.4 & 5.0 & $\mathrm{~N}$ & \\
\hline & 4 & -97.9 & $5.7(0.3)$ & 10.1 & 16.2 & 3.9 & $\mathrm{~N}$ & \\
\hline \multirow[t]{5}{*}{ G338.6347+00.1378 } & 1 & -27.4 & $2.4(0.7)$ & 13.4 & 5.8 & 6.3 & $\mathrm{~N}$ & \\
\hline & 2 & -45.9 & $3.5(0.5)$ & 12.3 & 8.4 & 5.4 & $\mathrm{~N}$ & \\
\hline & 3 & -80.5 & $5.0(0.3)$ & 10.8 & 12.0 & 4.2 & $\mathrm{~N} ?$ & strongest? \\
\hline & 4 & -60.5 & $4.2(0.4)$ & 11.6 & 10.1 & 4.8 & $\mathrm{~N}$ & \\
\hline & 5 & -35.0 & $2.9(0.6)$ & 13.0 & 7.0 & 5.9 & $\mathrm{~N}$ & \\
\hline \multirow[t]{3}{*}{ G339.4938-00.7986 } & 1 & -34.0 & $2.9(0.6)$ & 13.1 & -40.4 & 5.9 & $\mathrm{~N}$ & strongest? \\
\hline & 2 & -23.6 & $2.2(0.7)$ & 13.8 & -30.7 & 6.5 & $\mathrm{~N}$ & \\
\hline & 3 & -43.4 & 3.4 & $12.5(0.5)$ & -174.2 & 5.4 & $\mathrm{~F}$ & \\
\hline \multirow[t]{7}{*}{ G339.6221-00.1209 } & 1 & -33.7 & $2.9(0.6)$ & 13.1 & -6.1 & 5.9 & $\mathrm{~N}$ & \\
\hline & 2 & -22.1 & $2.1(0.8)$ & 13.9 & -4.4 & 6.6 & $\mathrm{~N}$ & \\
\hline & 3 & -93.5 & 5.5 & $10.4(0.3)$ & -21.9 & 3.8 & $\mathrm{~F}$ & \\
\hline & 4 & -120.8 & 6.5 & $9.4(0.3)$ & -19.8 & 3.3 & $\mathrm{~F}$ & \\
\hline & 5 & -43.3 & 3.4 & $12.5(0.5)$ & -26.4 & 5.4 & $\mathrm{~F}$ & \\
\hline & 6 & -8.8 & 0.9 & $15.0(1.0)$ & -31.7 & 7.6 & $\mathrm{~F}$ & \\
\hline & 7 & -70.7 & 4.7 & $11.3(0.4)$ & -23.8 & 4.4 & $\mathrm{~F}$ & \\
\hline G339.6816-01.2058 & & -28.0 & 2.5 & $13.4(0.7)$ & -282.0 & 6.2 & $\mathrm{~F} ?$ & \\
\hline \multirow{3}{*}{ G339.8712-00.6701 } & 1 & -91.8 & 5.5 & $10.5(0.3)$ & -122.8 & 3.8 & $\mathrm{~F} ?$ & strongest? \\
\hline & 2 & -21.5 & $2.0(0.8)$ & 13.9 & -23.4 & 6.6 & $\mathrm{~N}$ & \\
\hline & 3 & -34.7 & $2.9(0.6)$ & 13.0 & -33.9 & 5.8 & $\mathrm{~N}$ & \\
\hline \multirow[t]{3}{*}{ G339.8941-00.3419 } & 1 & -48.2 & $3.7(0.5)$ & 12.3 & -22.1 & 5.2 & $\mathrm{~N}$ & \\
\hline & 2 & -27.6 & 2.5 & $13.5(0.7)$ & -80.6 & 6.2 & $\mathrm{~F} ?$ & \\
\hline & 3 & -36.7 & $3.1(0.6)$ & 12.9 & -18.5 & 5.7 & $\mathrm{~N}$ & \\
\hline \multirow[t]{2}{*}{ G340.1412-01.0532 } & 1 & -28.8 & $2.6(0.7)$ & 13.4 & -47.8 & 6.1 & $\mathrm{~N} ?$ & SA/off-beam? \\
\hline & 2 & -41.4 & 3.3 & $12.6(0.5)$ & -231.6 & 5.5 & $\mathrm{~F}$ & \\
\hline \multirow[t]{2}{*}{ G340.1537+00.5116 } & 1 & -52.7 & 3.9 & $12.1(0.5)$ & 108.0 & 5.0 & $\mathrm{~F} ?$ & \\
\hline & 2 & -127.8 & 6.8 & $9.2(0.3)$ & 82.1 & 3.1 & $\mathrm{~F}$ & \\
\hline \multirow[t]{2}{*}{ G340.2637-01.3236 } & 1 & -18.6 & $1.8(0.8)$ & 14.2 & -41.6 & 6.8 & $\mathrm{~N} ?$ & \\
\hline & 2 & -27.8 & 2.5 & $13.5(0.7)$ & -311.9 & 6.2 & $\mathrm{~F} ?$ & \\
\hline \multirow[t]{3}{*}{ G340.3314-00.2733 } & 1 & -46.6 & $3.6(0.5)$ & 12.4 & -17.2 & 5.2 & $\mathrm{~N}$ & Double?/SA \\
\hline & 2 & -53.0 & 4.0 & $12.0(0.5)$ & -57.2 & 5.0 & $\mathrm{~F}$ & \\
\hline & 3 & -37.2 & 3.1 & $12.9(0.6)$ & -61.5 & 5.7 & $\mathrm{~F}$ & \\
\hline \multirow[t]{3}{*}{ G340.5454-00.3754 } & 1 & -46.9 & 3.7 & $12.4(0.5)$ & -81.2 & 5.2 & $\mathrm{~F}$ & structure \\
\hline & 2 & -28.2 & $2.6(0.7)$ & 13.5 & -17.0 & 6.1 & $\mathrm{~N} ?$ & \\
\hline & 3 & -90.3 & 5.5 & $10.6(0.3)$ & -69.5 & 3.8 & $\mathrm{~F}$ & \\
\hline G341.1281-00.3466 & & -41.3 & $3.4(0.6)$ & 12.7 & -20.6 & 5.4 & $\mathrm{~N}$ & \\
\hline \multirow[t]{2}{*}{ G342.2263-00.3801 } & 1 & -25.9 & $2.5(0.8)$ & 13.7 & -16.6 & 6.1 & $\mathrm{~N} ?$ & \\
\hline & 2 & -43.6 & $3.6(0.5)$ & 12.5 & -23.9 & 5.2 & $\mathrm{~N}$ & \\
\hline G342.3877+00.0742 & 1 & -125.8 & 6.7 & $9.5(0.3)$ & 12.3 & 2.9 & $\mathrm{~F}$ & strongest? \\
\hline & 2 & -31.8 & $3.0(0.7)$ & 13.2 & 3.9 & 5.8 & $\mathrm{~N} ?$ & \\
\hline & 3 & -25.9 & $2.5(0.8)$ & 13.7 & 3.2 & 6.1 & $\mathrm{~N}$ & \\
\hline & 4 & -93.8 & 5.7 & $10.5(0.3)$ & 13.6 & 3.5 & $\mathrm{~F}$ & \\
\hline & 5 & -69.5 & 4.8 & $11.4(0.4)$ & 14.8 & 4.2 & $\mathrm{~F}$ & \\
\hline & 6 & -77.5 & $5.1(0.3)$ & 11.1 & 6.6 & 3.9 & $\mathrm{~N} ?$ & \\
\hline & 7 & -84.8 & 5.4 & $10.8(0.3)$ & 14.0 & 3.7 & $\mathrm{~F}$ & \\
\hline & 8 & -51.4 & 4.1 & $12.1(0.5)$ & 15.7 & 4.8 & $\mathrm{~F}$ & \\
\hline & 9 & -6.3 & $0.8(1.2)$ & 15.4 & 1.0 & 7.8 & $\mathrm{~N} ?$ & \\
\hline
\end{tabular}


Table A1 - Continued.

\begin{tabular}{|c|c|c|c|c|c|c|c|c|}
\hline Source Name & & $\begin{array}{c}\mathrm{V}_{L S R} \\
\left(\mathrm{~km} \mathrm{~s}^{-1}\right)\end{array}$ & $\begin{array}{l}\mathrm{D}_{\text {near }} \\
(\mathrm{kpc})\end{array}$ & $\begin{array}{l}\mathrm{D}_{f a r} \\
(\mathrm{kpc})\end{array}$ & $\begin{array}{c}\mathrm{z} \\
(\mathrm{pc})\end{array}$ & $\begin{array}{c}\mathrm{R}_{g c} \\
(\mathrm{kpc})\end{array}$ & $\begin{array}{l}\text { Adopted } \\
\text { Distance }\end{array}$ & Notes \\
\hline \multirow[t]{4}{*}{ G342.3891-00.0723 } & 1 & -21.5 & $2.2(0.8)$ & 14.0 & -2.8 & 6.4 & $\mathrm{~N}$ & \\
\hline & 2 & -85.8 & 5.4 & $10.8(0.3)$ & -13.6 & 3.7 & $\mathrm{~F}$ & \\
\hline & 3 & -123.5 & 6.6 & $9.6(0.3)$ & -12.1 & 3.0 & $\mathrm{~F}$ & \\
\hline & 4 & -131.3 & 6.9 & $9.4(0.3)$ & -11.9 & 2.9 & $\mathrm{~F}$ & \\
\hline \multirow[t]{5}{*}{ G342.8089-00.3825 } & 1 & -122.3 & 6.6 & $9.7(0.2)$ & -64.8 & 3.0 & $\mathrm{~F}$ & \\
\hline & 2 & -4.8 & $0.6(1.3)$ & 15.6 & -4.0 & 7.9 & $\mathrm{~N}$ & \\
\hline & 3 & -39.6 & 3.5 & $12.8(0.6)$ & -85.5 & 5.3 & $\mathrm{~F}$ & \\
\hline & 4 & -27.5 & $2.7(0.7)$ & $13.5(0.7)$ & - & 6.0 & $?$ & ambiguous \\
\hline & 5 & -80.6 & 5.3 & $11.0(0.3)$ & -73.4 & 3.8 & $\mathrm{~F}$ & \\
\hline \multirow[t]{2}{*}{ G342.9583-00.3180 } & 1 & -39.7 & 3.5 & $12.8(0.6)$ & -71.0 & 5.3 & $\mathrm{~F}$ & \\
\hline & 2 & -30.0 & $2.9(0.7)$ & $13.4(0.7)$ & -16.1 & 5.8 & $\mathrm{~N} ?$ & \\
\hline G342.9636-00.3685 & & -127.3 & 6.7 & $9.5(0.2)$ & -61.1 & 2.9 & $\mathrm{~F}$ & \\
\hline \multirow[t]{4}{*}{ G343.1880-00.0803 } & 1 & -29.2 & $2.9(0.7)$ & 13.4 & -4.1 & 5.8 & $\mathrm{~N} ?$ & \\
\hline & 2 & -121.4 & $6.6(0.2)$ & 9.7 & -9.2 & 2.9 & $\mathrm{~N} ?$ & SA/off-beam? \\
\hline & 3 & -117.0 & 6.4 & $9.8(0.2)$ & -13.7 & 3.0 & $\mathrm{~F}$ & \\
\hline & 4 & -79.1 & $5.3(0.3)$ & $11.0(0.3)$ & - & 3.8 & $?$ & ambiguous \\
\hline \multirow[t]{2}{*}{ G343.5024-00.0145 } & 1 & -27.7 & $2.8(0.7)$ & 13.5 & -0.7 & 5.9 & $\mathrm{~N}$ & \\
\hline & 2 & -4.1 & $0.5(1.4)$ & 15.8 & -0.1 & 8.0 & $\mathrm{~N}$ & \\
\hline \multirow[t]{3}{*}{ G343.5213-00.5171 } & 1 & -34.9 & $3.3(0.6)$ & $13.0(0.6)$ & - & 5.5 & $?$ & ambiguous \\
\hline & 2 & -19.6 & $2.1(0.9)$ & $14.2(0.9)$ & - & 6.5 & $?$ & ambiguous \\
\hline & 3 & -78.6 & 5.3 & $11.0(0.3)$ & -99.3 & 3.8 & $\mathrm{~F}$ & \\
\hline \multirow[t]{2}{*}{ G343.5526+00.3705 } & 1 & -119.0 & $6.5(0.2)$ & 9.8 & 42.0 & 2.9 & $\mathrm{~N} ?$ & strongest? \\
\hline & 2 & -8.7 & 1.1 & $15.2(1.2)$ & 98.3 & 7.5 & $\mathrm{~F}$ & \\
\hline \multirow[t]{6}{*}{ G343.5928-00.1523 } & 1 & -27.3 & $2.8(0.8)$ & 13.5 & -7.4 & 5.9 & $\mathrm{~N}$ & SA/double? \\
\hline & 2 & -32.7 & 3.1 & $13.2(0.7)$ & -35.1 & 5.6 & $\mathrm{~F}$ & \\
\hline & 3 & -116.6 & 6.4 & $9.9(0.2)$ & -26.3 & 3.0 & $\mathrm{~F}$ & \\
\hline & 4 & -15.8 & 1.8 & $14.5(1.0)$ & -38.5 & 6.8 & $\mathrm{~F} ?$ & \\
\hline & 5 & -3.6 & $0.5(1.4)$ & 15.8 & -1.3 & 8.0 & $\mathrm{~N}$ & \\
\hline & 6 & -99.1 & 5.9 & $10.4(0.3)$ & -27.6 & 3.3 & $\mathrm{~F}$ & \\
\hline \multirow[t]{3}{*}{ G343.6489-00.1842 } & 1 & -27.4 & $2.8(0.8)$ & 13.5 & -9.0 & 5.9 & $\mathrm{~N}$ & SA/double? \\
\hline & 2 & -31.9 & $3.1(0.7)$ & 13.2 & -10.0 & 5.6 & $\mathrm{~N}$ & \\
\hline & 3 & -15.9 & 1.8 & $14.5(1.0)$ & -46.6 & 6.8 & $\mathrm{~F}$ & \\
\hline G343.7225+00.8327 & & -18.9 & $2.1(0.9)$ & 14.2 & 30.5 & 6.5 & $\mathrm{~N} ?$ & \\
\hline \multirow[t]{3}{*}{ G344.7291-00.5689 } & 1 & -25.7 & $2.8(0.8)$ & 13.6 & -27.8 & 5.9 & $\mathrm{~N}$ & strongest? \\
\hline & 2 & -17.6 & $2.1(1.0)$ & 14.3 & -20.9 & 6.5 & $\mathrm{~N}$ & \\
\hline & 3 & -32.9 & $3.3(0.7)$ & 13.1 & -32.8 & 5.4 & $\mathrm{~N}$ & \\
\hline G344.8889+01.4349 & & -13.4 & $1.7(1.1)$ & 14.7 & 42.6 & 6.9 & $\mathrm{~N} ?$ & \\
\hline G345.3307+01.4361 & & -14.2 & $1.8(1.1)$ & 14.6 & 45.1 & 6.8 & $\mathrm{~N} ?$ & \\
\hline G345.3766-00.9023 & & -21.2 & $2.5(0.9)$ & 14.0 & -39.4 & 6.2 & $\mathrm{~N}$ & \\
\hline \multirow[t]{2}{*}{ G345.5043+00.3480 } & 1 & -17.8 & $2.2(1.0)$ & 14.3 & 13.4 & 6.4 & $\mathrm{~N} ?$ & \\
\hline & 2 & -38.1 & $3.7(0.6)$ & 12.8 & 22.5 & 5.0 & $\mathrm{~N} ?$ & \\
\hline \multirow[t]{2}{*}{ G345.9561+00.6123 } & 1 & -11.4 & $1.5(1.3)$ & 14.9 & 16.0 & 7.0 & $\mathrm{~N}$ & strongest? \\
\hline & 2 & -21.9 & $2.6(0.9)$ & 13.9 & 27.8 & 6.0 & $\mathrm{~N}$ & \\
\hline G346.2331-00.3197 & & -11.5 & 1.6 & $14.9(1.3)$ & -83.1 & 7.0 & $\mathrm{~F} ?$ & \\
\hline \multirow{2}{*}{ G347.6327+00.2551 } & 1 & -85.7 & 5.9 & $10.7(0.3)$ & 47.6 & 3.0 & $\mathrm{~F}$ & structure \\
\hline & 2 & -73.4 & 5.5 & $11.1(0.3)$ & 49.4 & 3.3 & $\mathrm{~F}$ & \\
\hline \multirow[t]{2}{*}{ G347.8349+00.0849 } & 1 & -66.7 & 5.3 & $11.3(0.3)$ & 16.7 & 3.5 & $\mathrm{~F}$ & \\
\hline & 2 & -8.7 & 1.4 & $15.2(1.6)$ & 22.5 & 7.2 & $\mathrm{~F}$ & structure \\
\hline G347.8646+00.0189 & & -30.9 & 3.5 & $13.1(0.8)$ & 4.3 & 5.1 & $\mathrm{~F}$ & \\
\hline G347.8903-00.2796 & 1 & -93.3 & 6.1 & $10.5(0.2)$ & -51.2 & 2.8 & $\mathrm{~F}$ & \\
\hline & 2 & -21.0 & $2.7(1.0)$ & 13.9 & -13.2 & 5.9 & $\mathrm{~N} ?$ & \\
\hline G348.0489+00.2322 & 1 & -9.0 & $1.4(1.6)$ & 15.2 & 5.7 & 7.1 & $\mathrm{~N}$ & SA/double? \\
\hline & 2 & -12.1 & $1.8(1.4)$ & 14.8 & 7.3 & 6.7 & $\mathrm{~N}$ & \\
\hline & 3 & -0.9 & 0.2 & $16.5(2.3)$ & 66.9 & 8.3 & $\mathrm{~F} ?$ & \\
\hline & 4 & -88.2 & 6.0 & $10.6(0.2)$ & 43.0 & 2.9 & $\mathrm{~F}$ & strongest? \\
\hline G348.0679+00.2043 & 1 & -90.5 & 6.1 & $10.6(0.2)$ & 37.8 & 2.8 & $\mathrm{~F}$ & strongest? \\
\hline & 2 & -8.7 & $1.4(1.6)$ & 15.2 & 5.0 & 7.1 & $\mathrm{~N} ?$ & SA/off-beam? \\
\hline & 3 & -12.3 & $1.9(1.4)$ & 14.8 & 6.8 & 6.7 & $\mathrm{~N} ?$ & \\
\hline G348.2943+00.6365 & 1 & -8.0 & $1.3(1.7)$ & 15.3 & 14.4 & 7.2 & $\mathrm{~N}$ & \\
\hline & 2 & -28.8 & 3.5 & $13.2(0.8)$ & 146.6 & 5.2 & $\mathrm{~F}$ & \\
\hline
\end{tabular}


Table A1 - Continued.

\begin{tabular}{|c|c|c|c|c|c|c|c|c|}
\hline Source Name & & $\begin{array}{c}\mathrm{V}_{L S R} \\
\left(\mathrm{~km} \mathrm{~s}^{-1}\right)\end{array}$ & $\begin{array}{c}\mathrm{D}_{\text {near }} \\
(\mathrm{kpc})\end{array}$ & $\begin{array}{l}\mathrm{D}_{\text {far }} \\
(\mathrm{kpc})\end{array}$ & $\begin{array}{c}\mathrm{z} \\
(\mathrm{pc})\end{array}$ & $\begin{array}{c}\mathrm{R}_{g c} \\
(\mathrm{kpc})\end{array}$ & $\begin{array}{l}\text { Adopted } \\
\text { Distance }\end{array}$ & Notes \\
\hline G348.2949-00.5160 & & -19.5 & 2.7 & $14.0(1.1)$ & -126.1 & 5.9 & $\mathrm{~F}$ & \\
\hline \multirow[t]{2}{*}{ G348.5107-01.1199 } & 1 & -14.4 & 2.2 & $14.5(1.3)$ & -283.5 & 6.4 & $\mathrm{~F}$ & \\
\hline & 2 & -32.9 & 3.8 & $12.9(0.7)$ & -252.2 & 4.9 & $\mathrm{~F}$ & \\
\hline \multirow[t]{4}{*}{ G348.5547-00.9856 } & 1 & -14.7 & 2.2 & $14.5(1.3)$ & -249.5 & 6.4 & $\mathrm{~F}$ & \\
\hline & 2 & -6.1 & $1.1(1.9)$ & 15.6 & -18.9 & 7.5 & $\mathrm{~N}$ & \\
\hline & 3 & -29.2 & 3.5 & $13.1(0.8)$ & -225.4 & 5.1 & $\mathrm{~F}$ & SA/weak \\
\hline & 4 & -33.2 & 3.8 & $12.9(0.7)$ & -221.9 & 4.8 & $\mathrm{~F}$ & \\
\hline \multirow[t]{2}{*}{ G348.5625-00.9328 } & 1 & -13.7 & 2.1 & $14.6(1.4)$ & -237.7 & 6.5 & $\mathrm{~F}$ & $\mathrm{SA}$ \\
\hline & 2 & -33.6 & 3.8 & $12.8(0.7)$ & -208.4 & 4.8 & $\mathrm{~F}$ & weak \\
\hline \multirow[t]{3}{*}{ G348.7121+00.3279 } & 1 & -6.6 & $1.1(1.8)$ & 15.5 & 6.3 & 7.4 & $\mathrm{~N}$ & \\
\hline & 2 & -105.5 & $6.5(0.2)$ & 10.2 & 37.2 & 2.5 & $\mathrm{~N} ?$ & \\
\hline & 3 & -65.3 & 5.4 & $11.3(0.3)$ & 64.7 & 3.4 & $\mathrm{~F}$ & \\
\hline G348.7250-01.0435 & & -12.7 & $2.0(1.4)$ & 14.7 & -36.4 & 6.6 & $\mathrm{~N}$ & \\
\hline G348.8683-00.5534 & & -28.0 & 3.5 & $13.2(0.8)$ & -127.5 & 5.1 & $\mathrm{~F}$ & \\
\hline \multirow[t]{2}{*}{ G348.8922-00.1787 } & 1 & -98.4 & 6.4 & $10.3(0.2)$ & -32.1 & 2.6 & $\mathrm{~F}$ & \\
\hline & 2 & -118.3 & 6.8 & $9.9(0.2)$ & -30.9 & 2.2 & $\mathrm{~F}$ & \\
\hline \multirow[t]{2}{*}{ G349.0944+00.0807 } & 1 & -23.5 & $3.2(1.0)$ & 13.5 & 4.5 & 5.4 & $\mathrm{~N}$ & \\
\hline & 2 & -77.3 & 5.8 & $10.8(0.3)$ & 15.2 & 3.0 & $\mathrm{~F}$ & strongest? \\
\hline \multirow[t]{2}{*}{ G349.1772+00.0277 } & 1 & -77.9 & 5.9 & $10.8(0.3)$ & 5.2 & 2.9 & $\mathrm{~F}$ & strongest? \\
\hline & 2 & -97.9 & 6.4 & $10.3(0.2)$ & 5.0 & 2.5 & $\mathrm{~F}$ & \\
\hline \multirow[t]{3}{*}{ G349.2169+00.1450 } & 1 & -64.1 & 5.4 & $11.3(0.3)$ & 28.6 & 3.3 & $\mathrm{~F}$ & \\
\hline & 2 & -81.2 & 6.0 & $10.7(0.3)$ & 27.1 & 2.9 & $\mathrm{~F}$ & \\
\hline & 3 & -41.1 & 4.4 & $12.3(0.6)$ & 31.1 & 4.3 & $\mathrm{~F}$ & \\
\hline \multirow[t]{3}{*}{ G349.3514-00.2114 } & 1 & -26.7 & 6.1 & $10.6(0.9)$ & -39.1 & 2.8 & $\mathrm{~F}$ & strongest? \\
\hline & 2 & -13.8 & 2.2 & $14.5(1.4)$ & -53.5 & 6.4 & $\mathrm{~F}$ & off-beam \\
\hline & 3 & -83.6 & 3.5 & $13.2(0.2)$ & -48.7 & 5.1 & $\mathrm{~F}$ & \\
\hline G349.7948+00.9357 & & -4.3 & 0.9 & $15.9(2.3)$ & 259.7 & 7.7 & $\mathrm{~F}$ & \\
\hline G316.8119-00.0578 & & -39.5 & $2.8(0.6)$ & 9.6 & -2.8 & 6.7 & $\mathrm{~N} ?$ & \\
\hline G318.9476-00.1963 1 & & -36.1 & 2.5 & $10.3(0.6)$ & -35.3 & 6.8 & $\mathrm{~F} ?$ & \\
\hline G323.7408-00.2631 ${ }^{1}$ & & -51.5 & $3.4(0.5)$ & 10.3 & -15.6 & 6.1 & $\mathrm{~N}$ & \\
\hline G331.2788-00.1887 1 & & -90.5 & $5.4(0.4)$ & 9.5 & -17.8 & 4.6 & $\mathrm{~N}$ & \\
\hline G332.7250-00.6201 ${ }^{1}$ & & -50.6 & $3.4(0.5)$ & 11.7 & -36.8 & 5.7 & $\mathrm{~N} ?$ & \\
\hline
\end{tabular}

\footnotetext{
${ }^{1} \mathrm{~N}_{2} \mathrm{H}^{+}$maser line being used instead of ${ }^{13} \mathrm{CO}$ line.
}

The first column shows the $M S X$ object name in Galactic co-ordinates with any weaker line-of-sight components labeled 1-n. All coordinates are taken from the second release of the $M S X$ point source catalogue. The second column gives the $\mathrm{v}_{L S R}$ of the ${ }^{13} \mathrm{CO}$ component(s), or central $\mathrm{N}_{2} \mathrm{H}^{+}$masing line. Columns three and four are the calculated near and far distances using the standard Galactic rotation parameters $\left(R_{0}=8.5 \mathrm{kpc}, \Theta_{0}=220 \mathrm{~km} \mathrm{~s}^{-1}\right)$ of Brand \& Blitz (1993). If the kinematic distance ambiguity can be solved, the correct distance is labelled with bold font along with a typical $\pm 10 \mathrm{~km} \mathrm{~s}^{-1}$ peculiar velocity distance error. Columns five and six are the scale height and distance to the Galactic centre $\left(\mathrm{R}_{g c}\right)$ of the source or component respectively. The final column shows the adopted distance when compared to the H I data, where $\mathrm{N}$ and $\mathrm{F}$ represents near and far-distances respectively, with $\mathrm{N}$ ? and F? representing the most probable solution to slightly questionable spectra. A N? generally represents a dip in $\mathrm{H}_{\mathrm{I}}$ is present, but much less than 15-20 or is complicated via velocity structure in ${ }^{13} \mathrm{CO}$. A F? generally represents a dip may be present in $\mathrm{H}$ I but could be atributed to noise, or is shifted from the ${ }^{13} \mathrm{CO}$ peak position. A lone question mark denotes a still unsolved distance ambiguity. Any line profile problems are briefly noted in the final column. Notes index: SA/Off-beam/double sources have potential absorption at the chosen velocity component due to self absorption, off-beam objects, or could be considered to have double-peaked profiles. Structure indicates the line profile is not a typical gaussian. Strongest? represents a questionable strongest/best component line within the spectra which may or may not be the true potential MYSO from the MSX images. Ambiguous sources are those which still cannot be solved with this method 
Table A2. ${ }^{13} \mathrm{CO}$ 1-0 Kinematic parameters of $M S X$ sources geometrically solved with no distance ambiguity

\begin{tabular}{lccccccc}
\hline \hline \multicolumn{1}{c}{ Source Name } & $\begin{array}{c}\mathrm{V}_{L S R} \\
\left(\mathrm{~km} \mathrm{~s}^{-1}\right)\end{array}$ & $\begin{array}{c}\mathrm{D}_{\text {near }} \\
(\mathrm{kpc})\end{array}$ & $\begin{array}{c}\mathrm{D}_{\text {far }} \\
(\mathrm{kpc})\end{array}$ & $\begin{array}{c}\mathrm{z} \\
(\mathrm{pc})\end{array}$ & $\begin{array}{c}\mathrm{R}_{g c} \\
(\mathrm{kpc})\end{array}$ & $\begin{array}{c}\text { Adopted } \\
\text { Distance }\end{array}$ & Notes \\
\hline $\mathrm{G} 281.5961-00.1362$ & 20.8 & - & $6.1(1.0)$ & -14.5 & 9.4 & - & $\mathrm{n} / \mathrm{a}$ \\
$\mathrm{G} 282.2992-00.7763$ & 5.5 & - & $4.5(1.2)$ & -61.0 & 8.7 & - & $\mathrm{n} / \mathrm{a}$ \\
$\mathrm{G} 290.4756-00.3681$ & 18.8 & - & $7.9(0.9)$ & -50.8 & 9.4 & - & $\mathrm{n} / \mathrm{a}$ \\
$\mathrm{G} 295.4820-00.4464$ & 41.6 & - & $11.2(1.0)$ & -87.3 & 10.8 & - & $\mathrm{n} / \mathrm{a}$ \\
$\mathrm{G} 296.4038-01.0192$ & 27.6 & - & $10.1(0.9)$ & -179.7 & 9.9 & - & $\mathrm{n} / \mathrm{a}$ \\
$\mathrm{G} 303.9973+00.2800$ & 30.7 & - & $12.2(1.0)$ & 59.6 & 10.2 & - & $\mathrm{n} / \mathrm{a}$ \\
$\mathrm{G} 306.0951-00.4778$ & 41.7 & - & $13.7(1.1)$ & -114.2 & 11.1 & - & $\mathrm{n} / \mathrm{a}$ \\
$\mathrm{G} 306.1260-01.1371$ & 1.5 & - & $10.1(0.8)$ & -200.5 & 8.6 & - & $\mathrm{n} / \mathrm{a}$ \\
$\mathrm{G} 315.4195-00.3140$ & 4.5 & - & $12.5(0.8)$ & -68.5 & 8.8 & - & $\mathrm{n} / \mathrm{a}$ \\
$\mathrm{G} 325.5964+00.0912$ & 32.6 & - & $17.5(1.5)$ & 27.9 & 11.5 & - & $\mathrm{n} / \mathrm{a}$ \\
\hline
\end{tabular}

The first column shows the $M S X$ object name in Galactic co-ordinates. All co-ordinates are taken from the second release of the $M S X$ point source catalogue. The second column gives the $\mathrm{v}_{L S R}$ of the ${ }^{13} \mathrm{CO}$ component. Columns three and four are the calculated near and far distances using the standard Galactic rotation parameters $\left(R_{0}=8.5 \mathrm{kpc}, \Theta_{0}=220 \mathrm{~km} \mathrm{~s}^{-1}\right)$ of Brand \& Blitz (1993) with a typical $\pm 10 \mathrm{~km} \mathrm{~s}^{-1}$ peculiar velocity distance error. Columns five and six are the scale height and distance to the Galactic centre $\left(\mathrm{R}_{g c}\right)$ of the source. As these objects are geometrically solved, there are no near distance solutions given. 